\title{
Energy Efficiency Optimization for CoMP-SWIPT Heterogeneous Networks
}

DOI:

10.1109/TCOMM.2018.2866852

\section{Document Version}

Accepted author manuscript

Link to publication record in Manchester Research Explorer

\section{Citation for published version (APA):}

Tang, J., Shojaeifard, A., So, D. K. C., Wong, K., \& Zhao, N. (2018). Energy Efficiency Optimization for CoMPSWIPT Heterogeneous Networks. IEEE Transactions on Communications, 66(12), 6368-6383. https://doi.org/10.1109/TCOMM.2018.2866852

\section{Published in:}

IEEE Transactions on Communications

\section{Citing this paper}

Please note that where the full-text provided on Manchester Research Explorer is the Author Accepted Manuscript or Proof version this may differ from the final Published version. If citing, it is advised that you check and use the publisher's definitive version.

\section{General rights}

Copyright and moral rights for the publications made accessible in the Research Explorer are retained by the authors and/or other copyright owners and it is a condition of accessing publications that users recognise and abide by the legal requirements associated with these rights.

\section{Takedown policy}

If you believe that this document breaches copyright please refer to the University of Manchester's Takedown Procedures [http://man.ac.uk/04Y6Bo] or contact uml.scholarlycommunications@manchester.ac.uk providing relevant details, so we can investigate your claim.

\section{OPEN ACCESS}




\title{
Energy Efficiency Optimization for CoMP-SWIPT Heterogeneous Networks
}

\author{
Jie Tang, Senior Member, IEEE, Arman Shojaeifard, Member, IEEE, Daniel K. C. So, Senior Member, IEEE, \\ Kai-Kit Wong, Fellow, IEEE, and Nan Zhao, Senior Member, IEEE
}

\begin{abstract}
In this paper, a fundamental study of energy efficiency (EE) optimization for coordinated multi-point (CoMP)simultaneous wireless information and power transfer (SWIPT) heterogeneous networks (HetNets) is provided. We aim to optimize the EE whilst satisfying certain quality-of-service (QoS) requirements in regard to transmission rate and energy harvesting at both the macro-cell and small-cells. The corresponding joint beamforming and power allocation in the presence of intra- and inter-cell interference constitutes a EE maximization problem that is non-convex, and hence very challenging to solve. In order to solve this problem, we propose to separate the beamforming design and power allocation processes. First, we adopt linear zero-forcing ( $\mathrm{ZF}$ ) beamforming to suppress the multi-user interference from both the energy harvesting users (EH-UEs) as well as the information decoding users (ID-UEs), thus transforming the HetNet under consideration to a virtual point-to-point system. An efficient power allocation algorithm is then developed to maximize the corresponding EE. On the other hand, the ZF strategy does not utilize the notion that interference benefits the EH-UEs. As a result, we propose a partial zeroforcing (PZF) approach by differentiating the EH-UEs and IDUEs in order to further improve the EE. Our findings show that the EE can be significantly improved through the integration of CoMP-SWIPT in HetNets.
\end{abstract}

Index Terms-Coordinated multi-point (CoMP), energy efficiency (EE), heterogeneous networks (HetNets), simultaneous wireless information and power transfer (SWIPT).

\section{INTRODUCTION}

With the ever growing sales of smart devices, mobile data traffic has already surpassed voice traffic and continues to increase tremendously. To cater for this demand, the

This paper was presented in part at the IEEE/CIC International Conference on Communications in China (ICCC), Qingdao, China, 2017. This work has been supported in part by the National Natural Science Foundation of China under Grant 61601186 and 61871065 , in part by the Natural Science Foundation of Guangdong Province under Grant 2017A030313383, in part by the Guangzhou Science Technology and Innovation Commission under Grant 201707010159, in part by the open research fund of National Mobile Communications Research Laboratory, Southeast University (No. 2018D03), and in part by the Engineering and Physical Sciences Research Council of UK under Grant EP/N008219/1. (Corresponding author: Nan Zhao.)

J. Tang is with the School of Electronic and Information Engineering, South China University of Technology, Guangzhou and with the State Key Laboratory of Integrated Services Networks, Xidian University, China. (email: eejtang@scut.edu.cn).

D. K. C. So is with the School of Electrical and Electronic Engineering, University of Manchester, Manchester, United Kingdom. (e-mail: d.so@manchester.ac.uk).

A. Shojaeifard and K.-K Wong are with the Department of Electronic and Electrical Engineering, University College London, London, United Kingdom. (e-mail: a.shojaeifard@ucl.ac.uk; kai-kit.wong@ucl.ac.uk).

N. Zhao is with the School of Info. and Commun. Eng., Dalian University of Technology, Dalian 116024 China, and also with National Mobile Communications Research Laboratory, Southeast University, Nanjing 210096, China (email: zhaonan@dlut.edu.cn). wireless industry has been developing new technologies and standards to accommodate higher data rates, known as $5 \mathrm{G}$ [1]. A key enabler for $5 \mathrm{G}$ is network densification, where various types of base stations (BSs) are deployed to support user equipments (UEs) with increased density and qualityof-service (QoS) requirements, a.k.a., heterogeneous network (HetNet) [2], [3]. On the other hand, the increased interference introduced through network densification in HetNets can limit the achievable spectral-efficiency (SE). A prominent strategy for tackling the issue of interference is coordinated multipoint (CoMP) transmission, where the system performance can be significantly improved by mitigating inter-cell interference [4]-[7]. In particular, a parallel iteratively weighted minimum mean square error (WMMSE) approach has been proposed in [6] to handle the interference in multiple-input multipleoutput (MIMO) interfering broadcast channel, and this has been extended to CoMP-based LTE-Advanced network in [7] where proportional-fair resource allocation approach has been considered. Further, with the anticipated novel overhauls in the traditional cellular architecture such to limit the data exchange between various transmitters, CoMP techniques may be significantly more suited for deployment in 5G networks than the existing $4 \mathrm{G}$ systems [8]. As a result, in addition to other emerging solutions such as device-to-device (D2D) communications, full-duplex radios, and millimeter-wave bands, CoMP-enabled HetNet can be viewed as a prominent enabling technology for 5G and beyond [9]-[11].

Meanwhile, energy-efficiency (EE) is nowadays widely recognized an essential criteria in system design, and thus has attracted great interest in cellular networks [12]-[15], and more recently HetNets [16]-[18]. In [16], the authors developed an efficient EE maximization solution for HetNets with underlay spectrum access. In [17], the authors considered the EE optimization problem for spectrum-sharing HetNets under constraints to protect the macro-cell UEs. In [18], the authors also studied the EE optimization problem in a spectrum-sharing HetNet, where the cross-tier interference mitigation and imperfect hybrid spectrum sensing were jointly considered. On the basis of these fundamental studies, the EE performance in CoMP-aided HetNets was investigated in [19] and [20]. In [19], zero-forcing (ZF)-based energy-efficient precoding was investigated. In particular, a novel subspace decomposition method was proposed in order to exploit the specific HetNet feature in which all UEs required fixed-rate services. The authors in [20] considered a more general case where joint beamforming design and power assignment were utilized towards maximizing the HetNet EE. 
Energy harvesting (EH), on the other hand, is widely considered a de facto solution towards enhancing the lifespan of energy-constrained wireless devices [21]. Based on that, a new technology named wireless power transfer (WPT) enables the transmitter to transfer energy to the receivers with the radio frequency (RF) signals [22], [23]. Thus, integrating RF-based EH capability in traditional wireless communication systems has emerged recently as an important research direction, namely, simultaneous wireless information and power transfer (SWIPT), where information decoding (ID) and EH are performed simultaneously. Based on this idea, the informationtheoretic study was first investigated in [24]. However, given that the receivers sensitivity for information and energy are fundamentally different, this approach was not practically feasible [25]. Motivated by this, two new receiver structures are developed where ID and EH are separated through time domain and power domain [26], [27]. In addition, rather than splitting the data and energy through time or power domain, a new approach named spatial switching (SS) was developed for a MIMO-SWIPT system where the eigen-channels are either assigned to ID or EH [28].

As a result of the high energy requirements of wireless devices, EE performance has become a central theme towards delivering on the requirements of $5 \mathrm{G}$ in terms of user experience. With the introduction of the fundamental studies on SWIPT, EE maximization has attracted great attention in the context of SWIPT recently [29]-[33]. Based on powersplitting (PS) receivers, authors in [29] propose an iterative power allocation algorithm to maximize the EE. With ZF precoding and PS receivers, a Lagrangian relaxation method coupled with Dinkelbach method has been proposed in [30] to address the EE maximization problem in multi-user MISO SWIPT systems. This has been extended to multi-cell multiuser downlink systems where a coordinated beamforming (CB) strategy has been developed [31]. Rather than switching through power domain, a joint antenna selection and spatial switching scheme has been provided for EE optimization in a MIMO SWIPT system [32]. In [33], authors maximize the information transmission efficiency (ITE) of ID UEs and energy harvesting efficiency (EHE) of EH UEs, respectively, with the QoS of all users, and investigate their relationship. An efficient algorithm based on mixed beamforming (MBF) has been proposed to solve the ITE and EHE problems. The results therein revealed that the SWIPT-based solution is capable of providing additional EE gain compared to conventional systems. Furthermore, it has been shown in [19] and [20] that CoMP is a de facto approach towards achieving high EE in emerging dense cellular networks. Therefore, given the inherent high potential of CoMP-enabled HetNets, it is worth investigating the underlying EE performance through introduction of SWIPT technology.

\section{A. Main Contributions}

Although the above studies are beneficial for understanding the theoretical bounds of SWIPT systems, there are certain challenges when it comes to implementing SWIPT technology in practice. For instance, power requirements/sensitivities for wireless information receivers and energy receivers are different and hence they are designed separately (e.g., ID receivers such as cellular and Wi-Fi mobile receivers often operate with a received power less than $-50 \mathrm{dBm}$, while an $\mathrm{EH}$ receiver for a low-power sensor requires a received power of $0.1 \mathrm{~mW}$ or $-10 \mathrm{dBm}$ for real-time operation [27]). Therefore, existing EH circuits cannot be used for receiving information. This motivates our work to study the EE optimization for CoMPSWIPT HetNet in a practical setting, where the BSs with multiple antennas simultaneously transmit to multiple singleantenna receivers with either information or energy, but not both at the same time. Specifically, two different types of UEs are considered in our CoMP-SWIPT two-tier HetNet: (i) ID-UEs, such as tablets, cell phones, and laptops, which may be located far from the corresponding BSs, and (ii) EHUEs, such as sensors and other low-power devices, which are located relatively close to the BSs. Under this setup, we consider joint beamforming and power allocation for QoSconstrained EE optimization in the downlink of CoMP-SWIPT two-tier HetNets, where minimum rate constraints for ID-UEs and minimum harvested energy constraints for EH-UEs are taken into consideration. Furthermore, different from the work in [33] where the impact of ID and EH on EE has been considered separately in a SWIPT-enabled HetNet (ITE or EHE optimization), in this work both the impact of ID as well as EH on EE has been considered jointly, and hence a more complete EE optimization problem has been studied. The main contributions of this paper can be summarized as follows.

- The joint beamforming and power allocation problem for maximizing EE in the presence of inter- and intra-cell interference is non-convex; as a result, the solution is nontrivial. In order to tackle this, we develop two solutions where the beamforming design and power allocation procedure is separated for possible implementation in practical CoMP-SWIPT systems.

- For the first proposed zero forcing (ZF)-based approach, we suppress all interference to both ID-UEs and EH-UEs, and hence the considered HetNet scenario is transformed to a virtual point to point system. An efficient power allocation policy is then developed to optimize the EE through striking a balance in the ratio of the sum rate, transmission power, and transferred energy.

- The proposed ZF-based solution may not be efficient for EH because beneficial interference has not been fully exploited, i.e., the energy can be scavenged from the interference. To further improve the EE, a partial zeroforcing (PZF) approach is thus proposed where the pros and cons of interference are carefully taken into account. Specifically, we differentiate the EH-UEs and ID-UEs into two systems, namely, the EH system and the ID system. We first construct the null spaces for the ID system where all interference to ID-UEs is canceled. On the other hand, for the EH system, the interference is preserved in order to fully exploit the RF energy. Then, a hybrid transmit beamforming is developed to optimally combine the column space of the constructed null spaces. The proposed PZF scheme only needs around half the 
transmit antennas compared to the ZF-based scheme, and hence proves the applicability to a large practical system.

- The proposed PZF scheme achieves a lower computational complexity compared to the WMMSE algorithm for $\mathrm{CB}$ in a MIMO broadcasting channel [6], and the proportional-fair resource allocation algorithm in a CoMP based LTE-A system [7], and hence it demonstrates the applicability of the proposed solution. More importantly, compared to the mixed beamformer (MBF)-based EE maximization scheme in [33] where the impact of EH or ID on EE has been considered separately, our proposed PZF-based solution jointly considers the impact of both ID and $\mathrm{EH}$ on EE, and hence obtains a superior EE versus the MBF strategy in [33]. Furthermore, simulation results also validate the proposed ZF-based and PZFbased approaches, and show that significant EE gains can be achieved compared to the system without EH capability, i.e., MIMO broadcast channels [6], CoMPbased HetNets [20]. These results illustrate that the EE can be improved considerably through integration of CoMP-SWIPT technology.

\section{B. Organization}

The rest of this paper is organized as follows. In Section II, the CoMP-SWIPT two-tier HetNet setup and the corresponding EE problem formulation are provided. In Section III, a ZF-based beamforming strategy is proposed to handle the complicated interference environment. In particular, the HetNet scenario is transformed to a virtual point-to-point system by canceling all the interference to both EH-UEs and ID-UEs, and an efficient resource allocation scheme is then developed for maximizing the EE. In Section IV, a PZF-based approach is proposed in order to further improve the EE. The results of the simulations are presented in Section V, and finally, conclusions are provided in Section VI.

\section{Preliminaries}

In this section, we first introduce the system model of the proposed CoMP-SWIPT two-tier HetNet, and then formulate the corresponding QoS-constrained EE optimization problem.

\section{A. System Model}

We consider the downlink of a CoMP-SWIPT two-tier HetNet comprising a macro-cell and $L$ small-cells, as shown in Fig. 1. It is assumed that only one BS is providing service in each cell. The set of cells is denoted using $\mathcal{L}=\{0,1,2, \cdots, L\}$, where indexes 0 and $\{1,2, \cdots, L\}$ correspond to the macro-cell and the small-cells, respectively. For the SWIPT setup, two types of UEs are considered. In particular, the UEs are classified into ID UEs which receive and decode data from the corresponding BS, and EH UEs which are capable of harvesting energy from RF signals. In particular, the EH-UEs only harvest energy because of their power imbalance with ID-UE. $\mathcal{K}_{l}^{I}$ and $\mathcal{K}_{l}^{E}$ respectively represent the set of ID-UEs connected to cell $l \in \mathcal{L}$ and the set of EH-UEs associated with cell $l \in \mathcal{L}$, and $\left|\mathcal{K}_{l}^{I}\right|=K_{l}^{I}$,

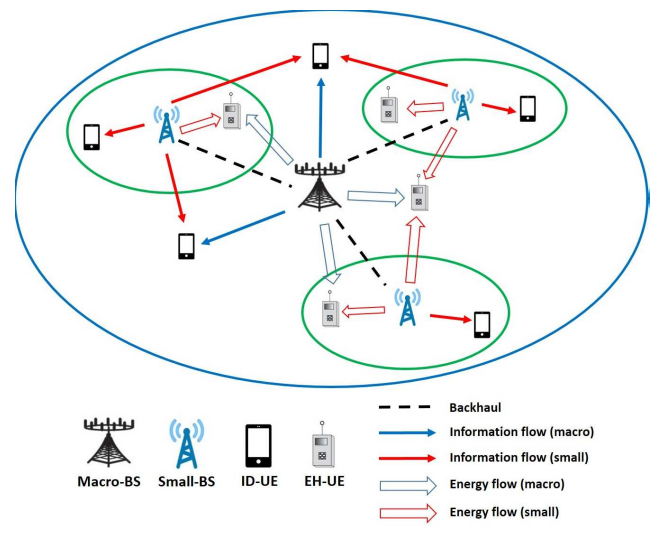

Fig. 1: A CoMP-SWIPT two-tier HetNet comprising of three smallcells and a macro-cell.

$\left|\mathcal{K}_{l}^{E}\right|=K_{l}^{E}$. The BS in cell $l \in \mathcal{L}$ is equipped with $N_{l}$ transmit antennas whilst a single antenna is employed for all ID-UEs and EH-UEs. CoMP joint processing (CoMP-JP) and CoMP-CB are two different approaches that can provide high SE in downlink HetNets. In particular, in order to convert the inter-cell interference (ICI) into useful signal, CoMP-JP is employed when there is sufficient backhaul among the coordinated BSs to share information. On the other hand, with a capacity-limited backhaul, which is not be able to support information sharing, CoMP-CB is used to avoid the ICI. In this paper, to emphasize the impact of heterogeneous deployment with SWIPT capabilities, similar to the work in [19], the backhaul between the macro-BS and the small-BSs is assumed to be capacity-sufficient, and hence CoMP-JP is employed. It is also assumed that perfect channel state information (CSI) is available at the BSs. For brevity, CoMP-JP is referred to as CoMP in the rest of the paper. Therefore, the transmitted signals at the BS $l$ are given by

$$
\mathbf{x}_{l}=\sum_{k \in \mathcal{K}_{l}^{I}} \mathbf{w}_{[k, l]} s_{[k, l]}^{I}+\sum_{k \in \mathcal{K}_{l}^{E}} \mathbf{v}_{[k, l]} s_{[k, l]}^{E},
$$

where $s_{[k, l]}^{I}$ and $\mathbf{w}_{[k, l]}$ respectively denote the data signal and its corresponding linear beamforming vector, $s_{[k, l]}^{E}$ and $\mathbf{v}_{[k, l]}$ are respectively representing the energy-carrying signal of EH$\mathrm{UE}[k, l]$ and its corresponding linear beamforming vector. In particular, we assume the data signal $s_{[k, l]}^{I}$ is independent and identically distributed (i.i.d) circularly symmetric complex Gaussian (CSCG) random variable with zero mean and unit variance. On the other hand, given that the energy signals carry no information, they can be modeled as random signals which meet a specific power spectral density. Therefore, $s_{[k, l]}^{E}$ is assumed to be an independent white sequence with $\mathbb{E}\left[\left|s_{[k, l]}^{E}\right|^{2}\right]=1$. Furthermore, the transmit beamforming vectors are written as

$$
\begin{aligned}
\mathbf{w}_{[k, l]} & =\sqrt{p_{[k, l]}} \overline{\mathbf{w}}_{[k, l]}, \\
\mathbf{v}_{[k, l]} & =\sqrt{q_{[k, l]}} \overline{\mathbf{v}}_{[k, l]},
\end{aligned}
$$

where $p_{[k, l]}$ and $q_{[k, l]}$ respectively denote the transmit power corresponding to the linear beamforming vector $\overline{\mathbf{w}}_{[k, l]}$ and 
$\overline{\mathbf{v}}_{[k, l]}$, and $\mathbb{E}\left[\left\|\overline{\mathbf{w}}_{[k, l]}\right\|^{2}\right]=\mathbb{E}\left[\| \overline{\mathbf{v}}_{[k, l]}||^{2}\right]=1$. The channel from the cell- $m$ BS to the $k$-th ID-UE in cell- $l$ is denoted as $\mathbf{h}_{[k, l]}^{m}$. Similarly, the channel from the cell- $m$ BS to the cell- $l k$-th EH-UE is denoted with $\mathbf{g}_{[k, l]}^{m}$. Note that channels are modeled as independent identically distributed quasi-static Rayleigh block fading. Hence, suppose the unintended data and energy signals are treated as interference, the interference received by ID-UE $[k, l]$ is

$$
\begin{gathered}
I_{[k, l]}=\sum_{j \in \mathcal{K}_{l}^{I} \backslash\{k\}}\left|\mathbf{w}_{[j, l]}^{H} \mathbf{h}_{[k, l]}^{l}\right|^{2}+\sum_{i \in \mathcal{L} \backslash\{l\}} \sum_{j \in \mathcal{K}_{i}^{I}}\left|\mathbf{w}_{[j, i]}^{H} \mathbf{h}_{[k, l]}^{i}\right|^{2} \\
+\sum_{i \in \mathcal{L}} \sum_{j \in \mathcal{K}_{i}^{E}}\left|\mathbf{v}_{[j, i]}^{H} \mathbf{h}_{[k, l]}^{i}\right|^{2} .
\end{gathered}
$$

Thus, the signal-to-interference-plus-noise ratio (SINR) at the ID-UE $[k, l]$ is given by

$$
\gamma_{[k, l]}=\frac{\left|\mathbf{w}_{[k, l]}^{H} \mathbf{h}_{[k, l]}^{l}\right|^{2}}{I_{[k, l]}+\sigma_{[k, l]}^{2}},
$$

where $\sigma_{[k, l]}^{2}$ is the noise power at the $k$-th ID-UE in cell- $l$. We thus can express the transmission rate for the $k$-th ID-UE in cell- $l$ using

$$
R_{[k, l]}=W \log _{2}\left(1+\gamma_{[k, l]}\right),
$$

where $W$ denotes the total available spectrum in the SWIPTbased HetNet.

The receiver with EH capability can harvest the energy from both data beams and energy beams, i.e., both w's and v's. Hence, the energy collected at the receiver of EH-UE $[k, l]$ is

$$
E_{[k, l]}=\eta\left(\sum_{i \in \mathcal{L}} \sum_{j \in \mathcal{K}_{i}^{I}}\left|\mathbf{w}_{[j, i]}^{H} \mathbf{g}_{[k, l]}^{i}\right|^{2}+\sum_{i \in \mathcal{L}} \sum_{j \in \mathcal{K}_{i}^{E}}\left|\mathbf{v}_{[j, i]}^{H} \mathbf{g}_{[k, l]}^{i}\right|^{2}\right),
$$

where $\eta$ indicates the loss when converting the energy.

For the considered CoMP-SWIPT HetNet system, the system's overall power consumption model should take into account the EH devices. In general, the current RF EH devices only consume small amounts of energy whilst the harvested energy compensates to the system total power consumption. Therefore, it opens up the possibility for the EE to be further improved through EH capabilities. Thus, similar to the power model used in [34], [35], we consider the impact of harvested power, and hence, the system total power consumption is given by

$$
P=\zeta P_{T}+P_{C}-E,
$$

where $\zeta$ accounts for the reciprocal of the power amplifier drain efficiency, $P_{T}=\sum_{l \in \mathcal{L}} \sum_{k \in \mathcal{K}_{l}^{I}} p_{[k, l]}+$ $\sum_{l \in \mathcal{L}} \sum_{k \in \mathcal{K}_{l}^{E}} q_{[k, l]}$ is the transmission power, $E=$ $\sum_{l \in \mathcal{L}} \sum_{k \in \mathcal{K}_{l}^{E}} E_{[k, l]}$ is the harvested power at all EH-UEs. It should be noted that the minus sign in (8) indicates that the receivers are capable of harvesting energy that is radiated in the RF from the transmitter. In addition, the circuit power consumption $P_{C}$ in our CoMP-SWIPT HetNet can be modelled as [36],

$$
P_{C}=\sum_{l \in \mathcal{L}}\left(P_{\text {ant }} N_{l}+P_{l}^{f i x}+P_{I} K_{l}^{I}+P_{E} K_{l}^{E}\right),
$$

where $P_{a n t} N_{l}$ represents the circuit power which depends on the number of transmit antennas at BS $l, P_{l}^{f i x}$ is the constant signal processing power at the $l^{\text {th }}$ cell. The last two terms $P_{I} K_{l}^{I}$ and $P_{E} K_{l}^{E}$ respectively denote the power required by all circuit components of each single-antenna ID-UE and EHUE.

\section{B. Problem Formulation}

The EE of our CoMP-SWIPT two-tier HetNet in the downlink can be formulated as (10) in the next page. Here, we are concerned with the problem of achieving maximum EE whilst meeting the required QoS constraints. Hence, the EE maximization problem should be formulated considering minimum transmission rate targets, minimum transferred energy demands, and total power budgets. Accordingly, the EE maximization problem for the CoMP-SWIPT HetNet is described as

$$
\begin{array}{ll}
\max _{\mathbf{w}_{[k, l]}, \mathbf{v}_{[k, l]}} & \lambda_{E E} \\
\text { s.t. } & \sum_{k \in \mathcal{K}_{l}^{I}}\left|\mathbf{w}_{[k, l]}\right|^{2}+\sum_{k \in \mathcal{K}_{l}^{E}}\left|\mathbf{v}_{[k, l]}\right|^{2} \leq P_{\text {max }}^{[l]}, \forall l \in \mathcal{L}, \\
& R_{[k, l]} \geq \delta_{[k, l]}, \quad \forall\{k, l\} \in \mathcal{K}_{l}^{I} \times \mathcal{L}, \\
& E_{[k, l]} \geq \rho_{[k, l]}, \forall\{k, l\} \in \mathcal{K}_{l}^{E} \times \mathcal{L},
\end{array}
$$

where $P_{\max }^{[l]}$ represents the transmit power budget for the cell$l, \delta_{[k, l]}$ represents the minimum rate target of ID-UE $[k, l]$ and $\rho_{[k, l]}$ denotes the minimum harvested energy for EH-UE $[k, l]$. Therefore, constraints (12)-(14) are respectively used to guarantee the maximum power budget, the minimum rate target and the minimum harvested energy requirement.

The EE optimization problem, with joint beamforming and power allocation in the presence of inter- and intra-cell interference, is non-convex. The solution is therefore nontrivial and cannot be obtained directly. In order to solve this EE optimization problem, one may rely on an exhaustive search method over all the possible beamforming and power allocation combinations. Nevertheless, it is obvious that this exhaustive search method incurs intensive computational complexity in the number of transmit antennas, UEs, and smallcells (which in practice can be very large). As a result, two different resource allocation strategies are developed with the help of ZF beamforming.

\section{Zero-Forcing Based Resource Allocation SCHEME}

In this section, we introduce the beamforming design and power allocation strategy for the proposed CoMP-SWIPT HetNet. We first consider the case where EH-UEs do not harvest the energy from the data signals of ID-UEs. A more general case where EH-UEs harvest all the existing signals will be considered in Section IV. Since the original problem in (11)-(14) is non-convex, it is non-trivial to derive the optimal beamforming directly, i.e., optimal $\mathbf{w}$ and $\mathbf{v}$. Even if we can develop an optimal joint beamforming and power allocation strategy, the computational complexity is often too high when considering a practical HetNet scenario 


$$
\lambda_{E E} \triangleq \frac{C}{P}=\frac{\sum_{l \in \mathcal{L}} \sum_{k \in \mathcal{K}_{l}^{I}} R_{[k, l]}}{\sum_{l \in \mathcal{L}}\left[\zeta\left(\sum_{k \in \mathcal{K}_{l}^{I}}\left|\mathbf{w}_{[k, l]}\right|^{2}+\sum_{k \in \mathcal{K}_{l}^{E}}\left|\mathbf{v}_{[k, l]}\right|^{2}\right)+P_{C}-\sum_{k \in \mathcal{K}_{l}^{E}} E_{[k, l]}\right]}
$$

$$
\begin{aligned}
& \mathbf{N}_{\left[k^{*}, l^{*}\right]}^{I} \subset \operatorname{null}\left(\left[\left(\mathbf{h}_{\left[k\left(k \in \mathcal{K}_{l}^{I}\right), l\left(l \in \mathcal{L} \backslash\left\{l^{*}\right\}\right)\right]}^{l^{*}}\right) \quad\left(\mathbf{h}_{\left[k\left(k \in \mathcal{K}_{l^{*}}^{I} \backslash\left\{k^{*}\right\}\right), l^{*}\right]}^{l^{*}}\right) \quad\left(\mathbf{g}_{\left[k\left(k \in \mathcal{K}_{l}^{E}\right), l(l \in \mathcal{L})\right]}^{l^{*}}\right)\right]^{H}\right) \\
& \mathbf{N}_{\left[k^{*}, l^{*}\right]}^{E} \subset \operatorname{null}\left(\left[\left(\mathbf{g}_{\left[k\left(k \in \mathcal{K}_{l}^{E}\right), l\left(l \in \mathcal{L} \backslash\left\{l^{*}\right\}\right)\right]}^{l^{*}}\right) \quad\left(\mathbf{g}_{\left[k\left(k \in \mathcal{K}_{l^{*}}^{E} \backslash\left\{k^{*}\right\}\right), l^{*}\right]}^{l^{*}}\right) \quad\left(\mathbf{h}_{\left[k\left(k \in \mathcal{K}_{l}^{I}\right), l(l \in \mathcal{L})\right]}^{l^{*}}\right)\right]^{H}\right)
\end{aligned}
$$

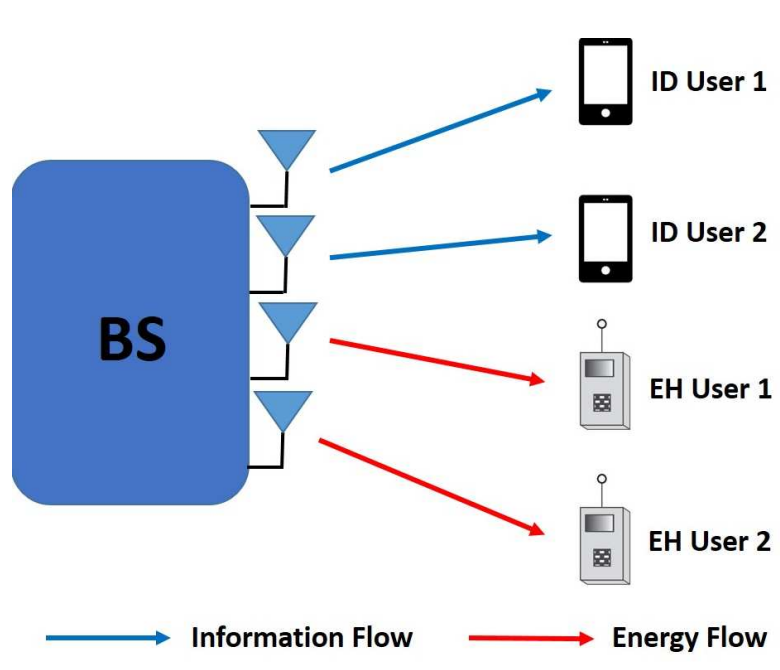

Fig. 2: Illustration of the signal space in CoMP-SWIPT HetNet with the $\mathrm{ZF}$ approach.

where there exists densely-populated small-cells and UEs. As a result, we separate the beamforming design and power allocation to facilitate possible implementation in practice.

The most common precoding and receive combining that are used in current wireless communication networks are $\mathrm{ZF}$, maximum ratio transmission/combining (MRT/MRC), and minimum mean squared error (MMSE) processing. However, it has been shown in [19] that ZF precoding provides the highest EE in a CoMP-enabled HetNet. Thus, ZF precoder is used here to remove the intra- and inter-cell interference jointly. In particular, we construct the null space as (15)(16) such that no interference is generated in the system. $\mathbf{H}_{\left[k \in \mathcal{K}_{l}^{I}, l \in \mathcal{L} \backslash\left\{l^{*}\right\}\right]}^{l^{*}}$ is a $N_{l^{*}} \times \sum_{l \in \mathcal{L} \backslash\left\{l^{*}\right\}} K_{l}^{I}$ matrix whose columns include the channels from the cell- $l^{*}$ BS to the ID-UEs in other cells $l \in \mathcal{L} \backslash\left\{l^{*}\right\} ; \mathbf{H}_{\left[k \in \mathcal{K}_{l^{*}}^{l^{*}} \backslash\left\{k^{*}\right\}, l^{*}\right]}$ is a $N_{l^{*}} \times\left(K_{l^{*}}^{I}-1\right)$ matrix whose columns are the channels from the cell- $l^{*}$ BS to its served ID-UEs; $\mathbf{G}_{\left.\left[k \in \mathcal{K}_{l}^{E}, l \in \mathcal{L}\right)\right]}^{l^{*}}$ is a $N_{l^{*}} \times \sum_{l \in \mathcal{L}} K_{l}^{E}$ matrix whose columns are the channels from the cell- $l^{*} \mathrm{BS}$ to all the EH-UEs. Therefore, the right hand size of (15) is a $N_{l^{*}} \times\left[\sum_{l \in \mathcal{L}}\left(K_{l}^{I}+K_{l}^{E}\right)-1\right]$ matrix, and the corresponding $\mathbf{N}_{\left[k^{*}, l^{*}\right]}^{I}$ is thus $N_{l^{*}} \times\left[N_{l^{*}}-\sum_{l \in \mathcal{L}}\left(K_{l}^{I}+K_{l}^{E}\right)+1\right]$ if the null space of (15) exists. Similarly, $\mathbf{N}_{\left[k^{*}, l^{*}\right]}^{E}$ is thus $N_{l^{*}} \times\left[N_{l^{*}}-\sum_{l \in \mathcal{L}}\left(K_{l}^{I}+K_{l}^{E}\right)+1\right]$ if the null space of (16) exists. Hence, we can use (15) and (16) to design the beamforming for all ID-UEs and EH-UEs in macro-cell and small-cells in the following way,

$$
\overline{\mathbf{w}}_{[k, l]}=\frac{\mathbf{N}_{[k, l]}^{I} \mathbf{N}_{[k, l]}^{I}{ }^{H} \mathbf{h}_{[k, l]}^{l}}{\left\|\mathbf{N}_{[k, l]}^{I} \mathbf{N}_{[k, l]}^{I}{ }^{H} \mathbf{h}_{[k, l]}^{l}\right\|}, \quad \forall\{k, l\} \in \mathcal{K}_{l}^{I} \times \mathcal{L}
$$

$$
\overline{\mathbf{v}}_{[k, l]}=\frac{\mathbf{N}_{[k, l]}^{E} \mathbf{N}_{[k, l]}^{E}{ }^{H} \mathbf{g}_{[k, l]}^{l}}{\left\|\mathbf{N}_{[k, l]}^{E} \mathbf{N}_{[k, l]}^{E}{ }^{H} \mathbf{g}_{[k, l]}^{l}\right\|}, \quad \forall\{k, l\} \in \mathcal{K}_{l}^{E} \times \mathcal{L}
$$

where $\mathbf{N}_{[k, l]}^{I}$ and $\mathbf{N}_{[k, l]}^{E}$ are the nullspace of (15) and (16) respectively. $\mathbf{h}_{[k, l]}^{l}$ and $\mathbf{g}_{[k, l]}^{l}$ are the channel from BS $l$ to its desired ID-UE and EH-UE respectively. Thus, the use of (17) and (18) can guarantee zero interference (i.e., $I_{[k, l]}=$ $0, \forall\{k, l\} \in \mathcal{K}_{l}^{I} \times \mathcal{L}$ ), and the corresponding system diagram is shown in Fig. 2. Moreover, the feasible minimum number of antennas in cell $l$ should be $N_{l}^{\text {min }}=\sum_{l \in \mathcal{L}}\left(K_{l}^{I}+K_{l}^{E}\right)$.

After designing the beamforming vectors for all the ID-UEs and EH-UEs, we need to perform power allocation towards maximizing the EE. Since the interference is completely cancelled by the ZF beamforming, the CoMP-SWIPT HetNet scenario has been transformed to a 'virtual' single-user model. Therefore, the corresponding effective channel power gains are

$$
\tilde{h}_{[k, l]}=\frac{\left(\overline{\mathbf{w}}_{[k, l]}^{H} \mathbf{h}_{[k, l]}^{l}\right)^{2}}{\sigma_{[k, l]}^{2}}
$$

and

$$
\tilde{g}_{[k, l]}=\frac{\left(\overline{\mathbf{v}}_{[k, l]}^{H} \mathbf{g}_{[k, l]}^{l}\right)^{2}}{\sigma_{[k, l]}^{2}} .
$$

Considering static power consumption $P_{l}^{s t a}=P_{l}^{f i x}+P_{I} K_{l}^{I}+$ $P_{E} K_{l}^{E}$, and substituting (17)-(20) in (11)-(14), the original optimization problem is given by

$$
\begin{aligned}
\max _{p_{[k, l]}, q_{[k, l]}} & \frac{\sum_{l \in \mathcal{L}} \sum_{k \in \mathcal{K}_{l}^{I}} W \log _{2}\left(1+p_{[k, l]} \tilde{h}_{[k, l]}\right)}{P} \\
\text { s.t. } & \sum_{k \in \mathcal{K}_{l}^{I}} p_{[k, l]}+\sum_{k \in \mathcal{K}_{l}^{E}} q_{[k, l]} \leq P_{\text {max }}^{[l]}, \quad \forall l \in \mathcal{L} \\
& W \log _{2}\left(1+p_{[k, l]} \tilde{h}_{[k, l]}\right) \geq \delta_{[k, l]}, \forall\{k, l\} \in \mathcal{K}_{l}^{I} \times \mathcal{L}, \\
& \eta q_{[k, l]} \tilde{g}_{[k, l]} \geq \rho_{[k, l]}, \quad \forall\{k, l\} \in \mathcal{K}_{l}^{E} \times \mathcal{L} .
\end{aligned}
$$

where $P=\sum_{l \in \mathcal{L}}\left[\zeta\left(\sum_{k \in \mathcal{K}_{l}^{I}} p_{[k, l]}+\sum_{k \in \mathcal{K}_{l}^{E}} q_{[k, l]}\right)+P_{\text {ant }} N_{l}+\right.$ $P_{l}^{s t a}-\sum_{k \in \mathcal{K}_{l}^{E}} \eta q_{[k, l]} \tilde{g}_{[k, l]]}$.

With ZF beamforming, the original problem in (11)-(14) is now transformed to the above power allocation problem. However, the objective function in (21) is a non-linear function, and the variables for power allocation, i.e., $p_{[k, l]}$ and $q_{[k, l]}$, are coupled together by the maximum power constraint; hence, the above non-convex problem cannot be solved trivially. To overcome this, we provide a fundamental study of energyefficient design in CoMP-SWIPT HetNets. In particular, a relationship between optimal $\mathrm{EE}$ and the transmit power $P_{T}$ is derived as in the following proposition.

Proposition 1: If there exists $E H-U E\{k, l\} \in \mathcal{K}_{l}^{E} \times \mathcal{L}$ 
satisfying $\eta \tilde{g}_{[k, l]}>\zeta$, the maximum $E E \lambda_{E E}^{*}$ is non-decreasing over the transmit power $P_{T}\left(P_{0} \leq P_{T} \leq P_{\max }\right)$, where $P_{0}$ is the minimum transmit power that satisfies the rate constraints in (23) and the EH constraints in (24).

Proof: Please see Appendix A.

Corollary: Proposition 1 further reveals that the maximum $E E \lambda_{E E}^{*}$ is achieved when $\sum_{k \in \mathcal{K}_{l}^{I}} p_{[k, l]}+\sum_{k \in \mathcal{K}_{l}^{E}} q_{[k, l]}=$ $P_{\text {max }}^{[l]}, \forall l \in \mathcal{L}$. However, if there does not exist any EH-UE $\{k, l\} \in \mathcal{K}_{l}^{E} \times \mathcal{L}$ that satisfies $\eta \tilde{g}_{[k, l]}>\zeta$, the most energyefficient strategy for all the EH-UEs is to meet the minimum harvested energy constraints with equality.

As a result, based on Proposition 1 and the corresponding Corollary, in what follows, we classify the solution into two cases.

1) Case 1: If there exists EH-UE $\{k, l\} \in \mathcal{K}_{l}^{E} \times \mathcal{L}$ satisfying $\eta \tilde{g}_{[k, l]}>\zeta$ :

In this case, the corresponding optimization problem is identical to (21)-(24) with the exception that the inequality in (22) becomes an equality, i.e., $\sum_{k \in \mathcal{K}_{l}^{I}} p_{[k, l]}+\sum_{k \in \mathcal{K}_{l}^{E}} q_{[k, l]}=$ $P_{\max }^{[l]}, \forall l \in \mathcal{L}$. This transformed problem is non-convex, as it involves a non-linear fractional programming problem. In order to tackle this, we first define a new combined UE set $\mathcal{K}_{l}^{I E}$, where $\mathcal{K}_{l}^{I E}=\mathcal{K}_{l}^{I} \cup \mathcal{K}_{l}^{E}$ and $\left|\mathcal{K}_{l}^{I E}\right|=K_{l}^{I}+K_{l}^{E}$. Specifically, the first $K_{l}^{I}$ UEs in $\mathcal{K}_{l}^{I E}$ are ID-UEs, whilst the remaining users are EH-UEs. In addition, we introduce the following symbols,

$$
\alpha_{[k, l]}= \begin{cases}1, & \{k, l\} \in \mathcal{K}_{l}^{I} \times \mathcal{L}, \\ 0, & \{k, l\} \in \mathcal{K}_{l}^{E} \times \mathcal{L},\end{cases}
$$

and the effective channel gain is reconstructed as

$$
\bar{h}_{[k, l]}= \begin{cases}\tilde{h}_{[k, l]}, & \{k, l\} \in \mathcal{K}_{l}^{I} \times \mathcal{L}, \\ \tilde{g}_{[k, l]}, & \{k, l\} \in \mathcal{K}_{l}^{E} \times \mathcal{L} .\end{cases}
$$

Thus, defining the transmit power for $\mathrm{UE}[k, l] \in\left\{\mathcal{K}_{l}^{I E}, \mathcal{L}\right\}$ as $p_{[k, l]}$, the transformed optimization problem where inequality in (22) has been updated to equality, can be reformulated as

$$
\begin{gathered}
\max _{p_{[k, l] \geq 0}} \frac{\sum_{l \in \mathcal{L}} \sum_{k \in \mathcal{K}_{l}^{I E}} W \log _{2}\left(1+\alpha_{[k, l]} p_{[k, l]} \bar{h}_{[k, l]}\right)}{\bar{P}} \\
\text { s.t. } \sum_{k \in \mathcal{K}_{l}^{I E}} p_{[k, l]}=P_{\text {max }}^{[l]}, \quad \forall l \in \mathcal{L}, \\
W \log _{2}\left(1+\alpha_{[k, l]} p_{[k, l]} \bar{h}_{[k, l]} \geq \delta_{[k, l]}, \quad \forall\{k, l\} \in \mathcal{K}_{l}^{I E} \times \mathcal{L},\right. \\
\eta\left(1-\alpha_{[k, l]}\right) p_{[k, l]} \bar{h}_{[k, l]} \geq \rho_{[k, l]}, \quad \forall\{k, l\} \in \mathcal{K}_{l}^{I E} \times \mathcal{L},
\end{gathered}
$$

where $\bar{P}=\sum_{l \in \mathcal{L}}\left[\zeta \sum_{k \in \mathcal{K}_{l}^{I E}} p_{[k, l]}+P_{a n t} N_{l}+P_{l}^{\text {sta }}-\right.$ $\left.\sum_{k \in \mathcal{K}_{l}^{I E}} \eta\left(1-\alpha_{[k, l]}\right) p_{[k, l]} \bar{h}_{[k, l]}\right]$, and it can be solved based on the following proposition.

Proposition 2: With power allocation $p_{[k, l]} \geq 0$, that meets the constraints in (28)-(30), the maximum EE, $\lambda_{E E}^{*}=$ $\max _{\left[p_{[k, l]}>0\right.} \lambda_{E E}\left(p_{[k, l]}\right)$, is strictly quasi-concave with respect to $p_{[k, l]}$.

Proof: Please see Appendix B.

We can write the Lagrangian function corresponding to problem (27)-(30) as (31) in the next page, where $\xi_{l} \geq 0$, $\varrho_{[k, l]} \geq 0$ and $\kappa_{[k, l]} \geq 0$ are the Lagrangian multipliers with respect to maximum power per cell, minimum rate per IDUE, and minimum transferred energy per EH-UE, respectively. Therefore, the corresponding dual objective function can be formulated as

$$
l\left(\xi_{l}, \varrho_{[k, l]}, \kappa_{[k, l]}\right)=\max _{p_{[k, l]} \geq 0} G\left(p_{[k, l]}, \xi_{l}, \varrho_{[k, l]}, \kappa_{[k, l]}\right) .
$$

Therefore, the dual problem is accordingly given by

$$
\min _{\xi_{l}, \varrho_{[k, l]}, \kappa_{[k, l]}} l\left(\xi_{l}, \varrho_{[k, l]}, \kappa_{[k, l]}\right) \text { s.t. } \xi_{l} \geq 0, \varrho_{[k, l]} \geq 0, \kappa_{[k, l]} \geq 0 .
$$

To obtain the optimal power allocation $p_{[k, l]}$, we can apply the gradient ascent method proposed in [37]. Specifically, we can approach $p_{[k, l]}$ sequentially with respect to its gradient direction of the Lagrangian function (31) in each iteration as (34) in the next page and

$$
p_{[k, l]}(n)=\left[p_{[k, l]}(n-1)+\varepsilon(n-1) \nabla_{p_{[k, l]}} G\right]^{+},
$$

where $\varepsilon$ represents the step size, $n\left(n \in\left\{1,2, \cdots, I_{\max }\right\}\right)$ denotes the iteration number with $I_{\max }$ being the maximum allowed number of iterations. In particular, the update of the step size should satisfy the following condition

$$
\sum_{n=1}^{\infty} \varepsilon(n)=\infty, \quad \sum_{n=1}^{\infty} \varepsilon(n)^{2}<\infty .
$$

After obtaining the power allocation $p_{[k, l]}^{*}$ using the gradient approach, i.e., (34) and (35), the corresponding optimal dual variables $\xi_{l}, \varrho_{[k, l]}, \kappa_{[k, l]}$ can be determined accordingly. It is easy to see that the Lagrangian function $l\left(\xi_{l}, \varrho_{[k, l]}, \kappa_{[k, l]}\right)$ is convex with respect to the dual variables $\xi_{l}, \varrho_{[k, l]}, \kappa_{[k, l]}$, thus we can apply one-dimensional searching scheme. However, since $l\left(\xi_{l}, \varrho_{[k, l]}, \kappa_{[k, l]}\right)$ is not guaranteed to be differentiable, this gradient method may not be applicable for some cases. Alternatively, we can use the well-known sub-gradient approach to approach the Lagrangian dual variables $\xi_{l}, \varrho_{[k, l]}, \kappa_{[k, l]}$, where sub-gradient direction is summarized as the following Proposition.

Proposition 3. $P_{\max }^{[l]}-\sum_{k \in \mathcal{K}_{l}^{I E}} p_{[k, l]}, \quad W \log _{2}(1+$ $\left.\alpha_{[k, l]} p_{[k, l]} \bar{h}_{[k, l]}\right)-\delta_{[k, l]}$ and $\eta\left(1-\alpha_{[k, l]}\right) p_{[k, l]} \bar{h}_{[k, l]}-$ $\rho_{[k, l]}$ are the subgradient of the dual objective function $l\left(\xi_{l}, \varrho_{[k, l]}, \kappa_{[k, l]}\right)$, respectively.

Proof: Please refer to [37] for details.

With the results in Proposition 3, we update the Lagrangian dual variables as

$$
\begin{gathered}
\xi_{l}(n)=\left[\xi_{l}(n-1)\right. \\
\left.+\bar{\varepsilon}(n-1)\left(\sum_{k \in \mathcal{K}_{l}^{I E}} p_{[k, l]}-P_{m a x}^{[l]}\right)\right]^{+}, \\
\varrho_{[k, l]}(n)=\left[\varrho_{[k, l]}(n-1)\right. \\
+\tilde{\varepsilon}(n-1)\left(\delta_{[k, l]}-W \log _{2}\left(\left(1+\alpha_{[k, l]} p_{[k, l]} \bar{h}_{[k, l]}\right)\right)\right]^{+}, \\
\kappa_{[k, l]}(n)=\left[\kappa_{[k, l]}(n-1)\right. \\
+\hat{\varepsilon}(n-1)\left(\rho_{[k, l]}-\eta\left(\left(1-\alpha_{[k, l]}\right) p_{[k, l]} \bar{h}_{[k, l]}\right)\right]^{+},
\end{gathered}
$$

and $\bar{\varepsilon}, \tilde{\varepsilon}$ and $\hat{\varepsilon}$ represents the aforementioned step size. 


$$
\begin{gathered}
G\left(p_{[k, l]}, \xi_{l}, \varrho_{[k, l]}, \kappa_{[k, l]}\right)=\frac{\sum_{l \in \mathcal{L}} \sum_{k \in \mathcal{K}_{l}^{I E}} W \log _{2}\left(1+\alpha_{[k, l]} p_{[k, l]} \bar{h}_{[k, l]}\right)}{\bar{P}}+\sum_{l \in \mathcal{L}} \xi_{l}\left(P_{m a x}^{[l]}-\sum_{k \in \mathcal{K}_{l}^{I E}} p_{[k, l]}\right) \\
+\sum_{l \in \mathcal{L}} \sum_{k \in \mathcal{K}_{l}^{I E}} \varrho_{[k, l]}\left[W \log _{2}\left(1+\alpha_{[k, l]} p_{[k, l]} \bar{h}_{[k, l]}\right)-\delta_{[k, l]}\right]+\sum_{l \in \mathcal{L}} \sum_{k \in \mathcal{K}_{l}^{I E}} \kappa_{[k, l]}\left[\eta\left(1-\alpha_{[k, l]}\right) p_{[k, l]} \bar{h}_{[k, l]}-\rho_{[k, l]}\right] \\
\nabla_{p_{[k, l]}} G:=\frac{\left(W \alpha_{[k, l]} \bar{h}_{[k, l]} \log _{2} e\right) /\left(1+\alpha_{[k, l]} p_{[k, l]} \bar{h}_{[k, l]}\right)}{\sum_{l \in \mathcal{L}}\left[\zeta \sum_{k \in \mathcal{K}_{l}^{I E}} p_{[k, l]}+P_{a n t} N_{l}+P_{l}^{s t a}-\sum_{k \in \mathcal{K}_{l}^{I E}} \eta\left(1-\alpha_{[k, l]}\right) p_{[k, l]} \bar{h}_{[k, l]}\right]}+\kappa_{[k, l]} \eta\left(1-\alpha_{[k, l]}\right) \bar{h}_{[k, l]} \\
-\frac{\left[\zeta-\eta\left(1-\alpha_{[k, l]}\right) \bar{h}_{[k, l]}\right] \sum_{l \in \mathcal{L}} \sum_{k \in \mathcal{K}_{l}^{I E}} W \log _{2}\left(1+\alpha_{[k, l]} p_{[k, l]} \bar{h}_{[k, l]}\right)}{\left(\sum_{l \in \mathcal{L}}\left[\zeta P_{\max }^{[l]}+P_{a n t} N_{l}+P_{l}^{s t a}-\sum_{k \in \mathcal{K}_{l}^{I E}} \eta\left(1-\alpha_{[k, l]}\right) p_{[k, l]} \bar{h}_{[k, l]}\right]\right)^{2}}-\xi_{l}+\frac{\varrho_{[k, l]} W \alpha_{[k, l]} \bar{h}_{[k, l]} \log _{2} e}{1+\alpha_{[k, l]} p_{[k, l]} \bar{h}_{[k, l]}}
\end{gathered}
$$

2) Case 2: All EH-UEs satisfy the condition $\eta \tilde{g}_{[k, l]}<\zeta$ :

Following from the statement in Corollary, if there does not exist any EH-UE $\{k, l\} \in \mathcal{K}_{l}^{E} \times \mathcal{L}$ that is satisfying $\eta \tilde{g}_{[k, l]}>\zeta$, the most energy-efficient strategy for all the EH-UEs is to meet the minimum harvest energy constraints with equality. Hence, the optimal power allocation for EH-UEs can be formulated as

$$
q_{[k, l]}^{*}=\frac{\rho_{[k, l]}}{\eta \tilde{g}_{[k, l]}} .
$$

With the optimal power allocation $q_{[k, l]}^{*}$, the original problem in (21)-(24) is given by

$$
\begin{aligned}
\max _{p_{[k, l]} \geq 0} & \frac{\sum_{l \in \mathcal{L}} \sum_{k \in \mathcal{K}_{l}^{I}} W \log _{2}\left(1+p_{[k, l]} \tilde{h}_{[k, l]}\right)}{\sum_{l \in \mathcal{L}}\left(\zeta \sum_{k \in \mathcal{K}_{l}^{I}} p_{[k, l]}+P_{l}^{f i x}\right)} \\
\text { s.t. } & \sum_{k \in \mathcal{K}_{l}^{I}} p_{[k, l]} \leq \bar{P}_{\text {max }}^{[l]}, \forall l \in \mathcal{L}, \\
& W \log _{2}\left(1+p_{[k, l]} \tilde{h}_{[k, l]}\right) \geq \delta_{[k, l]}, \quad \forall\{k, l\} \in \mathcal{K}_{l}^{I} \times \mathcal{L},
\end{aligned}
$$

where $P_{l}^{\text {fix }}=P_{\text {ant }} N_{l}+P_{l}^{s t a}-\sum_{k \in \mathcal{K}_{l}^{E}} \eta q_{[k, l]}^{*} \tilde{g}_{[k, l]}, \bar{P}_{\max }^{[l]}$ denotes the remaining power budget for cell $l$ and $\bar{P}_{\max }^{[l]}=$ $P_{\max }^{[l]}-\sum_{k \in \mathcal{K}_{l}^{E}} q_{[k, l]}^{*}$.

Proposition 4: With power allocation $p_{[k, l]} \geq 0$, that satisfies the constraints in (42)-(43), the maximum $E E, \lambda_{E E}^{*}=$ $\max _{p_{[k, l]}>0} \lambda_{E E}\left(p_{[k, l]}\right)$, is a strictly quasi-concave function in regard to $p_{[k, l]}$.

Proof: The proof is similar to that of Proposition 2, and hence is omitted here.

Therefore, similar to the solution of problem (27)-(30), we can obtain the optimal solution $p_{[k, l]}$ using the aforementioned gradient adaptation approach, and the corresponding dual variables are updated through the sub-gradient approach that is similar to (37)-(39).

\section{PZF-Based Resource Allocation Scheme}

The idea of the proposed ZF based solution is to cancel all the interference to both ID-UEs and EH-UEs, and hence it is efficient for data transmission. It is true that the interference is a degrading factor for all ID-UEs. However, interference is beneficial to all EH-UEs since they can collect energy from the surrounding environment. Therefore, to design the beamforming that is suitable for our CoMP-SWIPT HetNet scenario, we

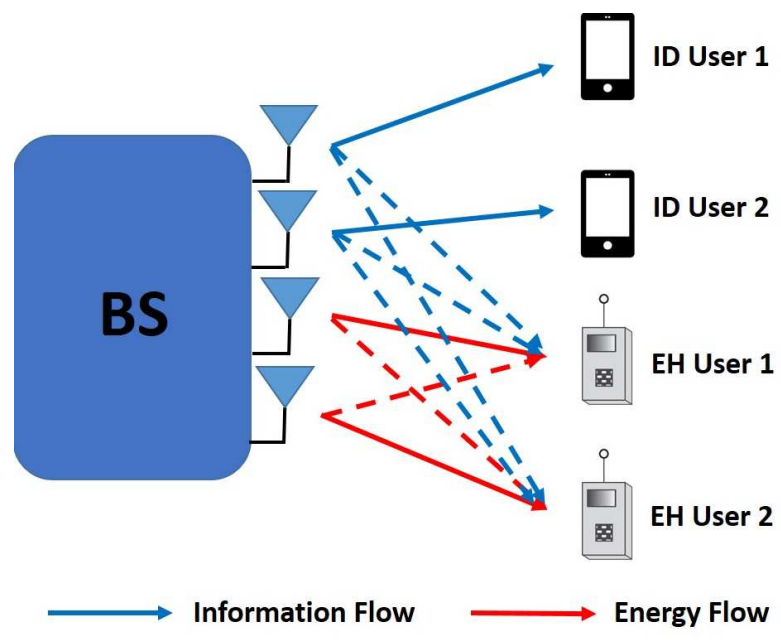

Fig. 3: Illustration of the signal space in CoMP-SWIPT HetNet with the PZF approach.

can differentiate between the EH-UEs and ID-UEs due to their different performance with respect to interference.

To fully exploit all existing interference, the ideal interference control strategy of the proposed CoMP-SWIPT is shown in Fig. 3. In particular, the precoder for the ID-UEs, $\overline{\mathbf{w}}_{[k, l]}, \forall\{k, l\} \in \mathcal{K}_{l}^{I} \times \mathcal{L}$, can be designed based on a similar ZF beamforming strategy proposed in Section III. On the other hand, we devise the EH precoder $\overline{\mathbf{v}}_{[k, l]}, \forall\{k, l\} \in \mathcal{K}_{l}^{E} \times \mathcal{L}$ with the fact that the interference is beneficial for all EH-UEs should be considered. Therefore, on the basis of interference impact of ID-UEs and EH-UEs, the design of the precoder for ID-UEs and EH-UEs should be perform separately.

We begin by constructing the following null space for ID$\mathrm{UE}\left[k^{*}, l^{*}\right]$,

$$
\overline{\mathbf{N}}_{\left[k^{*}, l^{*}\right]}^{I} \subset \operatorname{null}\left(\left[\left(\mathbf{H}_{\left[k \in \mathcal{K}_{l}^{I}, l \in \mathcal{L} \backslash\left\{l^{*}\right\}\right]}^{l^{*}}\right) \quad\left(\mathbf{H}_{\left[k \in \mathcal{K}_{l^{*}}^{I} \backslash\left\{k^{*}\right\}, l^{*}\right]}^{l^{*}}\right)\right]^{H}\right) .
$$

The right hand size of (44) is a $N_{l^{*}} \times\left(\sum_{l \in \mathcal{L}} K_{l}^{I}-1\right)$ matrix, and the corresponding $\overline{\mathbf{N}}_{\left[k^{*}, l^{*}\right]}^{I}$ is thus $N_{l^{*}} \times\left(N_{l^{*}}-\sum_{l \in \mathcal{L}} K_{l}^{I}+\right.$ $1)$ if the null space exists. Based on the constructed null space, the hybrid beamforming for ID-UE $\left[k^{*}, l^{*}\right]$, which consists of a PZF precoder, is written as

$$
\mathbf{w}_{\left[k^{*}, l^{*}\right]}=\overline{\mathbf{N}}_{\left[k^{*}, l^{*}\right]}^{I} \tilde{\mathbf{w}}_{\left[k^{*}, l^{*}\right]},
$$




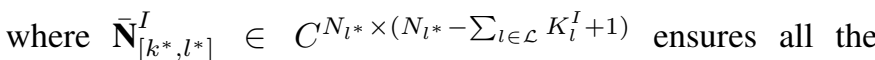
interference for ID-UEs is removed, and $\tilde{\mathbf{w}}_{\left[k^{*}, l^{*}\right]} \in$ $C^{\left(N_{l^{*}}-\sum_{l \in \mathcal{L}} K_{l}^{I}+1\right) \times 1}$ is used to exploit the column space of $\overline{\mathbf{N}}_{\left[k^{*}, l^{*}\right]}^{I}$ in order to further improve EE. In other words, the hybrid transmit beamforming $\mathbf{w}_{\left[k^{*}, l^{*}\right]}$ is capable of cancelling interference to ID-UEs whilst maximizing EE.

On the other hand, to fully exploit the interference for $\mathrm{EH}$ UEs, we construct the null space for EH-UEs in the cell $\left[l^{*}\right]$ as follows,

$$
\overline{\mathbf{N}}_{\left[l^{*}\right]}^{E} \subset \operatorname{null}\left(\left[\mathbf{H}_{\left[k \in \mathcal{K}_{l}^{I}, l \in \mathcal{L}\right]}^{l^{*}}\right]^{H}\right),
$$

such that the energy-carrying signal transmitted from BS $l^{*}$ does not generate interference to all the ID-UEs, where the right hand size of (46) is a $N_{l *} \times \sum_{l \in \mathcal{L}} K_{l}^{I}$ matrix, and the corresponding $\overline{\mathbf{N}}_{\left[k^{*}, l^{*}\right]}^{I}$ is thus $N_{l^{*}} \times\left(N_{l^{*}}-\sum_{l \in \mathcal{L}} K_{l}^{I}\right)$ if the null space of (46) exists. It should be noted that these null spaces for EH-UEs are shared within the cell, i.e., $\overline{\mathbf{N}}_{\left[l^{*}\right]}^{E}=\overline{\mathbf{N}}_{\left[1, l^{*}\right]}^{E}=\cdots=\overline{\mathbf{N}}_{\left[K_{L}^{E}, l^{*}\right]}^{E}$. Hence, we can write the hybrid beamforming for EH-UE $\left[k^{*}, l^{*}\right]$ based on the above constructed null space, which is given by

$$
\mathbf{v}_{\left[k^{*}, l^{*}\right]}=\overline{\mathbf{N}}_{\left[l^{*}\right]}^{E} \tilde{\mathbf{v}}_{\left[k^{*}, l^{*}\right]},
$$

where $\overline{\mathbf{N}}_{\left[l^{*}\right]}^{E} \in C^{N_{l^{*} \times} \times\left(N_{l^{*}}-\sum_{l \in \mathcal{L}} K_{l}^{I}\right)}$ represents the spaces that are shared by all the EH-UEs in the cell $l^{*}$. Similarly, $\tilde{\mathbf{v}}_{\left[k^{*}, l^{*}\right]} \in$ $C^{\left(N_{l^{*}}-\sum_{l \in \mathcal{L}} K_{l}^{I}\right) \times 1}$ is a vector that combines the columns of $\overline{\mathbf{N}}_{\left[l^{*}\right]}^{E}$ towards optimizing the EE. The effective channels are then given by

$$
\begin{aligned}
a_{[k, l]}^{\left[k^{*}, l^{*}\right]} & =\overline{\mathbf{N}}_{\left[k^{*}, l^{*}\right]}^{I} \mathbf{h}_{[k, l]}^{l^{*}}, \\
b_{[k, l]}^{\left[k^{*}, l^{*}\right]} & =\overline{\mathbf{N}}_{\left[k^{*}, l^{*}\right]}^{I} \mathbf{g}_{[k, l]}^{l^{*}}, \\
c_{[k, l]}^{\left[k^{*}, l^{*}\right]} & =\overline{\mathbf{N}}_{\left[l^{*}\right]}^{E H} \mathbf{g}_{[k, l]}^{l^{*}},
\end{aligned}
$$

where $a_{[k, l]}^{\left[k^{*}, l^{*}\right]}$ represents the effective channel from BS $l^{*}$ to ID-UE $[k, l]$ with the proposed PZF beamformer of ID$\mathrm{UE}\left[k^{*}, l^{*}\right], b_{[k, l]}^{\left[k^{*}, l^{*}\right]}$ denotes the effective channel from BS $l *$ to EH-UE $[k, l]$ with the proposed PZF beamformer of ID$\mathrm{UE}\left[k^{*}, l^{*}\right]$, and $c_{[k, l]}^{\left[k^{*}, l^{*}\right]}$ indicates the effective channel from BS $l *$ to EH-UE $[k, l]$ with the proposed PZF beamformer of $\mathrm{EH}-\mathrm{UE}\left[k^{*}, l^{*}\right]$. Furthermore, the feasible minimum number of antennas in cell $l$ should be $N_{l}^{\text {min }}=1+\sum_{l \in \mathcal{L}} K_{l}^{I}$.

Hence, with the proposed hybrid beamforming, our target is to design the effective beamformers $\tilde{\mathbf{w}}_{\left[k^{*}, l^{*}\right]}$ and $\tilde{\mathbf{v}}_{\left[k^{*}, l^{*}\right]}$ such that the system EE is maximized. To achieve this, the original optimization problem in (11)-(14) is reformulated as

$$
\begin{aligned}
& \max _{\tilde{\mathbf{w}}_{[k, l]}, \tilde{\mathbf{v}}_{[k, l]}} \frac{\sum_{l \in \mathcal{L}} \sum_{k \in \mathcal{K}_{l}^{I}} W \log _{2}\left(1+\frac{\left|a_{[k, l]}^{[k, l] H} \tilde{\mathbf{w}}_{[k, l]}\right|^{2}}{\sigma_{[k, l]}^{2}}\right)}{\text { s.t. }} \sum_{k \in \mathcal{K}_{l}^{I}}\left|\tilde{\mathbf{w}}_{[k, l]}\right|^{2}+\sum_{k \in \mathcal{K}_{l}^{E}}\left|\tilde{\mathbf{w}}_{[v, l]}\right|^{2} \leq P_{\text {max }}^{[l]}, \forall l \in \mathcal{L}, \\
& \\
& W \log _{2}\left(1+\frac{\left|a_{[k, l]}^{[k, l] H} \tilde{\mathbf{w}}_{[k, l]}\right|^{2}}{\sigma_{[k, l]}^{2}}\right) \geq \delta_{[k, l]}, \forall\{k, l\} \in \mathcal{K}_{l}^{I} \times \mathcal{L}, \\
& \\
& \quad\left(\sum_{l^{*} \in \mathcal{L}} \sum_{k^{*} \in \mathcal{K}_{l}^{I}}\left|b_{[k, l]}^{\left[k^{*}, l^{*}\right] H} \tilde{\mathbf{w}}_{\left[k^{*}, l^{*}\right]}\right|^{2}\right.
\end{aligned}
$$

$$
\left.+\sum_{l^{*} \in \mathcal{L}} \sum_{k^{*} \in \mathcal{K}_{l^{*}}^{E}}\left|c_{[k, l]}^{\left[k^{*}, l^{*}\right] H} \tilde{\mathbf{v}}_{\left[k^{*}, l^{*}\right]}\right|^{2}\right) \geq \rho_{[k, l]}, \forall\{k, l\} \in\left\{\mathcal{K}_{l}^{E}, \mathcal{L}\right\},
$$

where $\tilde{P}=\sum_{l \in \mathcal{L}}\left[\zeta\left(\sum_{k \in \mathcal{K}_{l}^{I}}\left|\tilde{\mathbf{w}}_{[k, l]}\right|^{2}+\sum_{k \in \mathcal{K}_{l}^{E}}\left|\tilde{\mathbf{v}}_{[k, l]}\right|^{2}\right)\right]+$ $P_{f i x}-\sum_{l \in \mathcal{L}} \sum_{k \in \mathcal{K}_{l}^{E}} E_{[k, l]}, P_{\text {fix }}=\sum_{l \in \mathcal{L}}\left(P_{a n t} N_{l}+P_{l}^{s t a}\right)$. Unfortunately, the above problem is a non-convex non-linear fractional programming problem. The solution is therefore nontrivial and cannot be obtained directly.

To solve this problem, we first define the beamforming and the effective channels in matrix form, i.e., $\tilde{\mathbf{W}}_{[k, l]}=\tilde{\mathbf{w}}_{[k, l]} \tilde{\mathbf{w}}_{[k, l]}^{H}$, $\tilde{\mathbf{V}}_{[k, l]}=\tilde{\mathbf{v}}_{[k, l]} \tilde{\mathbf{v}}_{[k, l]}^{H}, \mathbf{A}_{[k, l]}^{\left[k^{*}, l^{*}\right]}=\frac{1}{\sigma_{[k, l]}^{2}} a_{[k, l]}^{\left[k^{*}, l^{*}\right]} a_{[k, l]}^{\left[k^{*}, l^{*}\right] H}, \mathbf{B}_{[k, l]}^{\left[k^{*}, l^{*}\right]}=$ $b_{[k, l]}^{\left[k^{*}, l^{*}\right]} b_{[k, l]}^{\left[k^{*}, l^{*}\right] H}, \quad \mathbf{C}_{[k, l]}^{\left[k^{*}, l^{*}\right]}=c_{[k, l]}^{\left[k^{*}, l^{*}\right]} c_{[k, l]}^{\left[k^{*}, l^{*}\right] H}$. In addition, it has been shown in [38] that the Dinkelbach method is an effective scheme to solve the classic non-convex non-linear fractional programming problems. Therefore, with the help of Dinkelbach method, the EE maximization problem in (51)-(54) can be solved by transforming the fractional-form function into a subtractive-form function that is summarized as follows.

Proposition 5: The maximum EE $\beta^{*}=\psi_{E E}^{*}$ is achieved on condition that

$$
\begin{aligned}
& \max _{\tilde{\mathbf{W}}_{[k, l]}, \tilde{\mathbf{V}}_{[k, l]}} U_{R}\left(\tilde{\mathbf{W}}_{[k, l]}, \tilde{\mathbf{V}}_{[k, l]}\right)-\beta^{*} U_{T}\left(\tilde{\mathbf{W}}_{[k, l]}, \tilde{\mathbf{V}}_{[k, l]}\right) \\
& =U_{R}\left(\tilde{\mathbf{W}}_{[k, l]}^{*}, \tilde{\mathbf{V}}_{[k, l]}^{*}\right)-\beta^{*} U_{T}\left(\tilde{\mathbf{W}}_{[k, l]}^{*}, \tilde{\mathbf{V}}_{[k, l]}^{*}\right)=0,
\end{aligned}
$$

for $U_{R}\left(\tilde{\mathbf{W}}_{[k, l]}, \tilde{\mathbf{V}}_{[k, l]}\right) \geq 0$ and $U_{T}\left(\tilde{\mathbf{W}}_{[k, l]}, \tilde{\mathbf{V}}_{[k, l]}\right) \geq 0$, where

$$
\begin{aligned}
U_{R}\left(\tilde{\mathbf{W}}_{[k, l]}, \tilde{\mathbf{V}}_{[k, l]}\right) & =\sum_{l \in \mathcal{L}} \sum_{k \in \mathcal{K}_{l}^{I}} W \log _{2}\left(1+\operatorname{tr}\left(\mathbf{A}_{[k, l]}^{[k, l]} \tilde{\mathbf{W}}_{[k, l]}\right),(56)\right. \\
U_{T}\left(\tilde{\mathbf{W}}_{[k, l]}, \tilde{\mathbf{V}}_{[k, l]}\right) & =\sum_{l \in \mathcal{L}}\left[\zeta\left(\sum_{k \in \mathcal{K}_{l}^{I}} \operatorname{tr}\left(\tilde{\mathbf{W}}_{[k, l]}\right)+\sum_{k \in \mathcal{K}_{l}^{E}} \operatorname{tr}\left(\tilde{\mathbf{V}}_{[k, l]}\right)\right)\right] \\
& +P_{f i x}-\sum_{l \in \mathcal{L}} \sum_{k \in \mathcal{K}_{l}^{E}} E_{[k, l]},
\end{aligned}
$$

and $\beta^{*}=\frac{U_{R}\left(\tilde{\mathbf{W}}_{[k, l]}^{*}, \tilde{\mathbf{V}}_{[k, l]}^{*}\right)}{U_{T}\left(\tilde{\mathbf{W}}_{[k, l]}^{*}, \tilde{\mathbf{V}}_{[k, l]}^{*}\right)}$.

Proof: The detailed proof is provided in [38].

Proposition 5 provides a sufficient and necessary condition for obtaining the optimal results. Specifically, the EE maximization problem in (51)-(54) can be solved by transforming the fractional-form objective function (51) into a subtractiveform function (e.g. $U_{R}\left(\tilde{\mathbf{W}}_{[k, l]}, \tilde{\mathbf{V}}_{[k, l]}\right)-\beta^{*} U_{T}\left(\tilde{\mathbf{W}}_{[k, l]}, \tilde{\mathbf{V}}_{[k, l]}\right)$ ) can be found such that both optimization problems are sharing the same solution. Moreover, Proposition 5 further reveals that the maximum $\mathrm{EE}$ is obtained if the equality condition (55) is satisfied. Thus, this equality condition can be employed here to verify the optimality of the solution. As a result, we propose an iterative approach for the equivalent numeratordenominator subtractive-form objective function while satisfying the optimal-conditions in Proposition 5, as summarized in Table I.

As detailed in Table I, the key step is to tackle the equivalent problem under a fixed $\beta$, i.e., step 3 in Table I,

$$
\max _{\tilde{\mathbf{W}}_{[k, l]}, \tilde{\mathbf{V}}_{[k, l]}} \sum_{l \in \mathcal{L}} \sum_{k \in \mathcal{K}_{l}^{I}} W \log _{2}\left(1+\operatorname{tr}\left(\mathbf{A}_{[k, l]}^{[k, l]} \tilde{\mathbf{W}}_{[k, l]}\right)\right.
$$


TABLE I: PROPOSED DINKELBACH-BASED SOLUTION

1) Initialize $\beta=0$ and the stopping criteria $\delta=1 e^{-3}$;

2) REPEAT

3) Under a fixed $\beta$, tackle problem (59)-(62) to obtain the beamforming $\left\{\tilde{\mathbf{W}}_{[k, l]}, \tilde{\mathbf{V}}_{[k, l]}\right\}$;

4) $\quad$ IF $U_{R}\left(\tilde{\mathbf{W}}_{[k, l]}, \tilde{\mathbf{V}}_{[k, l]}\right)-\beta U_{T}\left(\tilde{\mathbf{W}}_{[k, l]}, \tilde{\mathbf{V}}_{[k, l]}\right) \leq \delta$

5) Convergence $=$ TRUE;

6) $\quad$ RETURN $\left\{\tilde{\mathbf{W}}_{[k, l]}^{*}, \tilde{\mathbf{V}}_{[k, l]}^{*}\right\}=\left\{\tilde{\mathbf{W}}_{[k, l]}, \tilde{\mathbf{V}}_{[k, l]}\right\}$

ELSE and $\beta^{*}=\frac{U_{R}\left(\tilde{\mathbf{W}}_{[k, l]}, \tilde{\mathbf{V}}_{[k, l]}\right)}{U_{T}\left(\tilde{\mathbf{W}}_{[k, l]}, \tilde{\mathbf{V}}_{[k, l]}\right)} ;$

7)

Set $\beta=\frac{U_{R}\left(\tilde{\mathbf{W}}_{[k, l]}, \tilde{\mathbf{V}}_{[k, l]}\right)}{U_{T}\left(\tilde{\mathbf{W}}_{[k, l]}, \tilde{\mathbf{V}}_{[k, l]}\right)}$ and $n=n+1$,

8) $\begin{aligned} & \text { Set } \beta=\frac{U_{R}\left(\tilde{\mathbf{W}}_{[k, l]}, \tilde{\mathbf{V}}_{[k, l]}\right)}{U_{T}\left(\tilde{\mathbf{W}}_{[k, l]}, \tilde{\mathbf{V}}_{[k, l]}\right)} \\ & \text { Convergence = FALSE; }\end{aligned}$

9) END IF

10) UNTIL Convergence $=$ TRUE.

$$
\begin{aligned}
& -\beta\left\{\sum_{l \in \mathcal{L}}\left[\zeta\left(\sum_{k \in \mathcal{K}_{l}^{I}} \operatorname{tr}\left(\tilde{\mathbf{W}}_{[k, l]}\right)+\sum_{k \in \mathcal{K}_{l}^{E}} \operatorname{tr}\left(\tilde{\mathbf{V}}_{[k, l]}\right)\right)\right]\right. \\
& \left.+P_{\text {fix }}-\sum_{l \in \mathcal{L}} \sum_{k \in \mathcal{K}_{l}^{E}} E_{[k, l]}\right\} \\
& \text { s.t. } \sum_{k \in \mathcal{K}_{l}^{I}} \operatorname{tr}\left(\tilde{\mathbf{W}}_{[k, l]}\right)+\sum_{k \in \mathcal{K}_{l}^{E}} \operatorname{tr}\left(\tilde{\mathbf{V}}_{[k, l]}\right) \leq P_{\text {max }}^{[l]}, \quad \forall l \in \mathcal{L}, \quad(60) \\
& \\
& \quad W \log _{2}\left(1+\operatorname{tr}\left(\mathbf{A}_{[k, l]}^{[k, l]} \tilde{\mathbf{W}}_{[k, l]}\right) \geq \delta_{[k, l]}, \quad \forall\{k, l\} \in\left\{\mathcal{K}_{l}^{I}, \mathcal{L}\right\},\right.
\end{aligned}
$$

$$
\begin{gathered}
\eta\left[\sum_{l^{*} \in \mathcal{L}} \sum_{k^{*} \in \mathcal{K}_{l}^{I}} \operatorname{tr}\left(\mathbf{B}_{[k, l]}^{\left[k^{*}, l^{*}\right]} \tilde{\mathbf{W}}_{\left[k^{*}, l^{*}\right]}\right)\right. \\
\left.+\sum_{l^{*} \in \mathcal{L}} \sum_{k^{*} \in \mathcal{K}_{l^{*}}^{E}} \operatorname{tr}\left(\mathbf{C}_{[k, l]}^{\left[k^{*}, l^{*}\right]} \tilde{\mathbf{V}}_{\left[k^{*}, l^{*}\right]}\right)\right] \geq \rho_{[k, l]}, \forall\{k, l\} \in\left\{\mathcal{K}_{l}^{E}, \mathcal{L}\right\} .
\end{gathered}
$$

Since $\operatorname{tr}(\mathbf{X})$ is linear with $\mathbf{X}$, and $\log |\mathbf{X}|$ is a concave function [39], the optimization problem (59)-(62) is convex. As a result, similar to the proposed ZF solution in Section III, the optimal solution, i.e., $\tilde{\mathbf{W}}_{[k, l]}$ and $\tilde{\mathbf{V}}_{[k, l]}$, can be achieved with the help of the gradient approach. In particular, we update $\tilde{\mathbf{W}}_{[k, l]}$ and $\tilde{\mathbf{V}}_{[k, l]}$ consecutively as follows,

$$
\begin{aligned}
\tilde{\mathbf{W}}_{[k, l]}(n) & =\left[\tilde{\mathbf{W}}_{[k, l]}(n-1)+\varpi(n-1) \nabla_{\tilde{\mathbf{W}}_{[k, l]}} \tilde{G}\right]^{+} \\
\tilde{\mathbf{V}}_{[k, l]}(n) & =\left[\tilde{\mathbf{V}}_{[k, l]}(n-1)+\bar{\varpi}(n-1) \nabla_{\tilde{\mathbf{V}}_{[k, l]}} \tilde{G}\right]^{+}
\end{aligned}
$$

where $\varpi$ and $\bar{\varpi}$ represent the step size of iteration which meet a similar condition as (36), and $\tilde{G}$ is the corresponding Lagrangian function for problem (59)-(62) which is given by (65) in the next page, with the gradient as

$$
\begin{gathered}
\nabla_{\tilde{\mathbf{W}}_{[k, l]}} \tilde{G}=\frac{W\left(1+\tilde{\varrho}_{[k, l]}\right) \mathbf{A}_{[k, l]}^{[k, l]}}{1+\operatorname{tr}\left(\mathbf{A}_{[k, l]}^{[k, l]} \tilde{\mathbf{W}}_{[k, l]}\right)}-\left(\beta \zeta+\tilde{\xi}_{l}\right) \mathbf{I}+ \\
\left(\beta-\tilde{\kappa}_{[k, l]}\right) \eta \sum_{l^{*} \in \mathcal{L}} \sum_{k^{*} \in \mathcal{K}_{l}^{I}} \mathbf{B}_{\left[k^{*}, l^{*}\right]}^{[k, l]}, \\
\nabla_{\tilde{\mathbf{V}}_{[k, l]}} \tilde{G}=-\left(\beta \zeta+\tilde{\xi}_{l}\right) \mathbf{I}+\left(\beta-\tilde{\kappa}_{[k, l]}\right) \eta \sum_{l^{*} \in \mathcal{L}} \sum_{k^{*} \in \mathcal{K}_{l}^{E}} \mathbf{C}_{\left[k^{*}, l^{*}\right]}^{[k, l]} .
\end{gathered}
$$

Once the optimal $\tilde{\mathbf{W}}_{[k, l]}$ and $\tilde{\mathbf{V}}_{[k, l]}$ are obtained using (63) and (64), the corresponding optimal Lagrangian dual variables $\tilde{\xi}_{l}, \tilde{\varrho}_{[k, l]}, \tilde{\kappa}_{[k, l]}$ can be determined. Similar to the sub-gradient approach used in Section III, the optimal Lagrangian dual variables can be updated as follows,

$$
\begin{gathered}
\xi_{l}(n)=\left[\xi_{l}(n-1)\right. \\
\left.+\tilde{\varpi}(n-1)\left(P_{\text {max }}^{[l]}-\sum_{k \in \mathcal{K}_{l}^{I}} \operatorname{tr}\left(\tilde{\mathbf{W}}_{[k, l]}\right)-\sum_{k \in \mathcal{K}_{l}^{E}} \operatorname{tr}\left(\tilde{\mathbf{V}}_{[k, l]}\right)\right)\right]^{+} \\
\varrho_{[k, l]}(n)=\left[\varrho_{[k, l]}(n-1)\right. \\
\left.+\hat{\varpi}(n-1)\left(W \log _{2}\left(1+\operatorname{tr}\left(\mathbf{A}_{[k, l]}^{[k, l]} \tilde{\mathbf{W}}_{[k, l]}\right)\right)-\delta_{[k, l]}\right)\right]^{+}, \\
\kappa_{[k, l]}(n)=\left[\kappa_{[k, l]}(n-1)+\check{\varpi}(n-1)\left(E_{[k, l]}-\rho_{[k, l]}\right)\right]^{+},
\end{gathered}
$$

and $\tilde{\varpi}, \hat{\varpi}$ and $\check{\varpi}$ represents the step size that meets the maximum condition in (36).

Remark: In the classic work on the sum rate maximization [40]-[42], the covariance matrix of each user is the same as the single user water-filling covariance matrix in a point-topoint link, where multiuser interference is treated as noise. However, as stated in [37], for the equivalent sum rate maximization which has a similar structure as (59)-(62), the optimal solution using gradient method does not possess a water-filling structure, and hence our algorithm does not obey the waterfilling principle. In addition, since problem (59)-(62) is convex, the set of the corresponding first order conditions (Karush-Kuhn-Tucker (KKT) conditions) is valid, and a fixed point of the proposed algorithm exists which is a stationary solution. Therefore, the proposed algorithm converges to an optimal set of the transmit signal covariance matrices.

In the aforementioned analysis, only one macro $\mathrm{BS}$ is considered to describe the operation of the proposed algorithms with considering the impact of macro cell-tier (co-tier) interference. For the case of multi-macro cell scenario, we can combine the co-tier interference and cross-tier interference together as inter-cell interference as per the analysis from the previous section, and hence the optimization problem for multi-macro cell scenario can be equivalently expressed as the single-macro cell scenario. Accordingly, our analysis still holds in the case of two-tier HetNets with multi-macro cell. Consequently, by employing the ZF-based algorithm proposed or the PZF-based algorithm, the EE optimization problem in a multi-macro cell scenario can be solved efficiently, which demonstrates the applicability of the proposed algorithms to multi-macro cell settings.

\section{A. Complexity Analysis}

In this section, we compare the computational complexity of our algorithm with that of the WMMSE algorithm for CB [6], and the CoMP-based proportional-fair resource allocation algorithm [7]. For simplicity, let us assume that the number of ID-UEs and EH-UEs are the same in each cell, i.e., $K_{l}^{I}=K_{I}, K_{l}^{E}=K_{E}, l=1,2, \cdots, L$, and $K_{I}+K_{E}=K$. In addition, each $\mathrm{BS}$ is equipped with the same number of transmit antennas $N_{l}=N_{t}, l=1,2, \cdots, L$. Similar to the 


$$
\begin{aligned}
& \tilde{G}\left(\tilde{\mathbf{W}}_{[k, l]}, \tilde{\mathbf{V}}_{[k, l]}, \tilde{\xi}_{l}, \tilde{\varrho}_{[k, l]}, \tilde{\kappa}_{[k, l]}\right)=\sum_{l \in \mathcal{L}} \sum_{k \in \mathcal{K}_{l}^{I}} W \log _{2}\left(1+\operatorname{tr}\left(\mathbf{A}_{[k, l]}^{[k, l]} \tilde{\mathbf{W}}_{[k, l]}\right)-\beta\left\{\sum_{l \in \mathcal{L}}\left[\zeta\left(\sum_{k \in \mathcal{K}_{l}^{I}} \operatorname{tr}\left(\tilde{\mathbf{W}}_{[k, l]}\right)+\sum_{k \in \mathcal{K}_{l}^{E}} \operatorname{tr}\left(\tilde{\mathbf{V}}_{[k, l]}\right)\right)\right]\right.\right. \\
& \left.+P_{f i x}-\sum_{l \in \mathcal{L}} \sum_{k \in \mathcal{K}_{l}^{E}} E_{[k, l]}\right\}+\sum_{l \in \mathcal{L}} \tilde{\xi}_{l}\left(P_{m a x}^{[l]}-\sum_{k \in \mathcal{K}_{l}^{I}} \operatorname{tr}\left(\tilde{\mathbf{W}}_{[k, l]}\right)-\sum_{k \in \mathcal{K}_{l}^{E}} \operatorname{tr}\left(\tilde{\mathbf{V}}_{[k, l]}\right)\right)+\sum_{l \in \mathcal{L}} \sum_{k \in \mathcal{K}_{l}^{I}} \tilde{\varrho}_{[k, l]}\left[W \log _{2}\left(1+\operatorname{tr}\left(\mathbf{A}_{[k, l]}^{[k, l]} \tilde{\mathbf{W}}_{[k, l]}\right)-\delta_{[k, l]}\right]\right. \\
& +\sum_{l \in \mathcal{L}} \sum_{k \in \mathcal{K}_{l}^{E}} \tilde{\kappa}_{[k, l]}\left\{\eta\left[\sum_{l^{*} \in \mathcal{L}} \sum_{k^{*} \in \mathcal{K}_{l}^{I}} \operatorname{tr}\left(\mathbf{B}_{[k, l]}^{\left[k^{*}, l^{*}\right]} \tilde{\mathbf{W}}_{\left[k^{*}, l^{*}\right]}\right)+\sum_{l^{*} \in \mathcal{L}} \sum_{k^{*} \in \mathcal{K}_{l^{*}}^{E}} \operatorname{tr}\left(\mathbf{C}_{[k, l]}^{\left[k^{*}, l^{*}\right]} \tilde{\mathbf{V}}_{\left[k^{*}, l^{*}\right]}\right)\right]-\rho_{[k, l]}\right\}
\end{aligned}
$$

works in [6] and [7], we compare the algorithms by given per iteration complexity, where an iteration means one round of updating all users' beamforming or covariance matrices. Under these conditions, each iteration of the proposed PZF solution involves the computation of the transmit covariance matrices, i.e., $\tilde{\mathbf{W}}_{[k, l]}$ in (66) and $\tilde{\mathbf{V}}_{[k, l]}$ in (67). Consequently, the per-iteration complexity of the proposed PZF algorithm is upper-bounded by $O\left(L K_{I}\left(N_{t}-L K_{I}+1\right)^{3}+L^{2} K^{2}\left(N_{t}-\right.\right.$ $\left.\left.L K_{I}+1\right)^{2}\right)$. On the other hand, the per-iteration complexity of the WMMSE algorithm and the CoMP-based proportionalfair algorithm is $O\left(L^{2} K^{2} N_{t}+L^{2} K^{2} N_{t}^{2}+L^{2} K^{2} N_{t}^{3}+L K\right)$ and $O\left(L^{2} K^{2} N_{t}+L^{2} K^{2} N_{t}^{2}+L K^{2} N_{t}^{3}+L K\right)$, respectively. Therefore, the computational complexity of our proposed PZF algorithm is lower than the WMMSE algorithm [6] and the CoMP-based solution [7].

\section{Simulation Results}

TABLE II: LIST OF SIMULATION PARAMETERS

\begin{tabular}{|c|c|}
\hline Bandwidth, $W$ & $20 \mathrm{MHz}$ \\
\hline Number of small-cells, $L$ & 3 \\
\hline Number of transmit antennas at BS $l, N_{l}$ & 20 \\
\hline Number of ID-UEs in cell $l, K_{l}^{I}$ & 2 \\
\hline Number of EH-UEs in cell $l, K_{l}^{E}$ & 2 \\
\hline Maximum transmit power of macro-cell, $P_{\max }^{[0]}$ & $40 \mathrm{~W}$ \\
\hline Maximum transmit power of small-cell, $P_{\max }^{[l]}$ & $20 \mathrm{~W}$ \\
\hline Total noise power, $W \sigma^{2}$ & $-96 \mathrm{dBm}$ \\
\hline Fixed power consumption at the macro-BS $P_{0}^{f i x}$ & $20 \mathrm{~W}$ \\
\hline Fixed power consumption at the small-BS $P_{l}^{f i x}$ & $10 \mathrm{~W}$ \\
\hline Circuit components attached to each antenna $P_{a n t}$ & $1 \mathrm{~W}$ \\
\hline Power required of each single-antenna ID-UE $P_{I}$ & $1 \mathrm{~W}$ \\
\hline Power required of each single-antenna EH-UE $P_{E}$ & $1 \mathrm{~W}$ \\
\hline
\end{tabular}

In this section, simulation results are provided in order to validate the performance of the proposed ZF-based and PZFbased approaches in the CoMP-SWIPT HetNet. It is assumed that three uniformly-distributed small-cells are in the coverage area of a macro-cell, where the radius of the macro-cell and the small-cells are set to $250 \mathrm{~m}$ and $50 \mathrm{~m}$, respectively. In order to model the capability of EH devices, such as sensors and other low-power devices, in a practically-feasible way, all EH-UEs are considered to be located within $20 \mathrm{~m}$ to the corresponding BSs [43]. All the results are obtained from various random locations of the UEs with identical and independent Rayleigh block fading channels and Log-Normal shadowing with standard deviation of $8 \mathrm{~dB}$. For the path loss, we here apply the generic channel model where the path-loss is given by $\left(\frac{d_{0}}{d}\right)^{v}$, where $d$ and $d_{0}$ are respectively representing the distance between the transmitter and the receiver and the

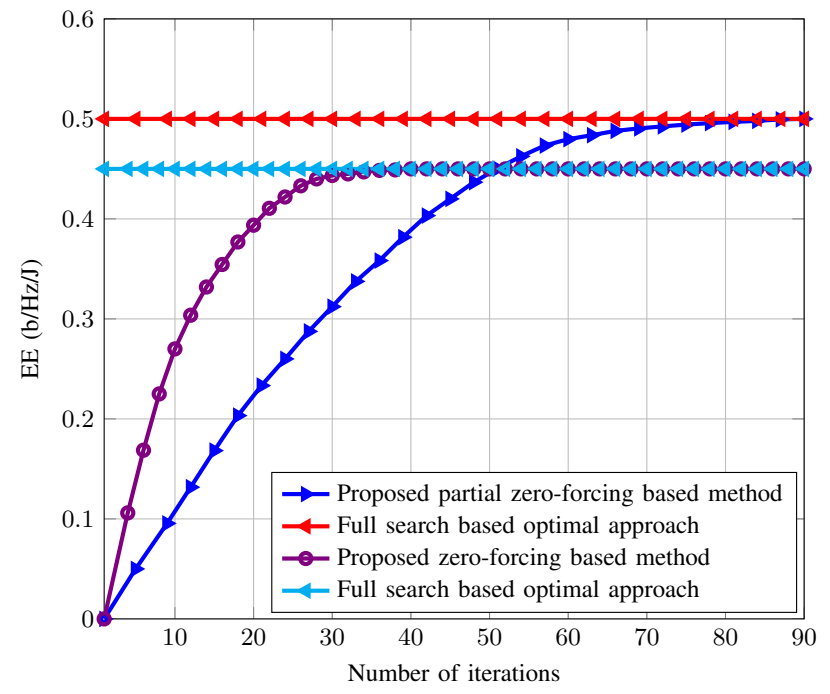

Fig. 4: Convergence behavior of the proposed ZF-based and PZFbased approaches.

reference distance, and the path-loss exponent $v$ is set to 2.5 and 3.76 respectively for a practical line-of-sight SWIPT scenario [44] and a typical 3GPP propagation environment [45]. The power amplifier drain efficiency $\zeta$ and the EH efficiency are set to $30 \%$ and $\eta=10 \%$, respectively [43]. In order to provide different service priorities and guaranteed QoS for each UE, the minimum rate constraint per ID-UE $\delta_{[k, l]}$ is set to $2 \mathrm{~b} / \mathrm{s} / \mathrm{Hz}$, and the minimum harvested energy per $\mathrm{EH}-$ UE $\rho_{[k, l]}$ is set to $0.2 \mathrm{~W}$ [43]. Other system parameters are defined in Table II.

First, we investigate the convergence behavior of the proposed ZF-based and PZF-based schemes. It can be seen from Fig. 4 that both algorithms are successfully converging to the optimal solution. Specifically, the EE solution is converging after around 40 and 90 iterations for the proposed ZF and PZF schemes, respectively. This result is in line with our theoretical analysis where the proposed PZF approach involves a more complicated joint beamforming design and power allocation procedure versus the proposed ZF scheme that only considers power allocation policy. Furthermore, as expected, the proposed PZF based approach outperforms the proposed ZF based scheme in terms of EE (approximately 11\%). This is because the former approach aims to fully utilize the beneficial interference for EH purposes without weakening the quality of data transmission, and hence, a higher EE is achieved.

Then, we evaluate the EE performance of the proposed ZF-based and PZF-based schemes under different scenario 


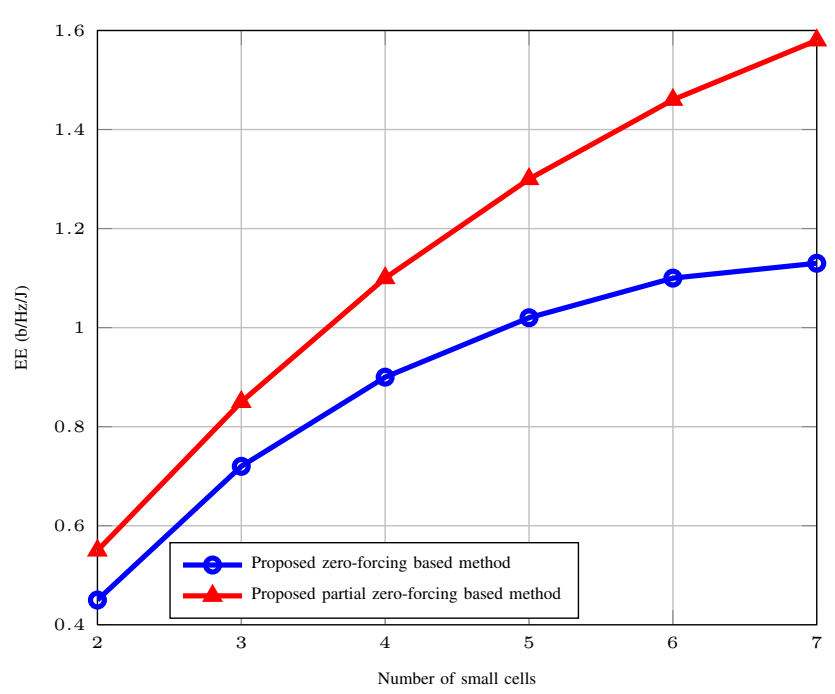

Fig. 5: Performance of the proposed ZF- and PZF-based approaches (EE vs number of small-cells).

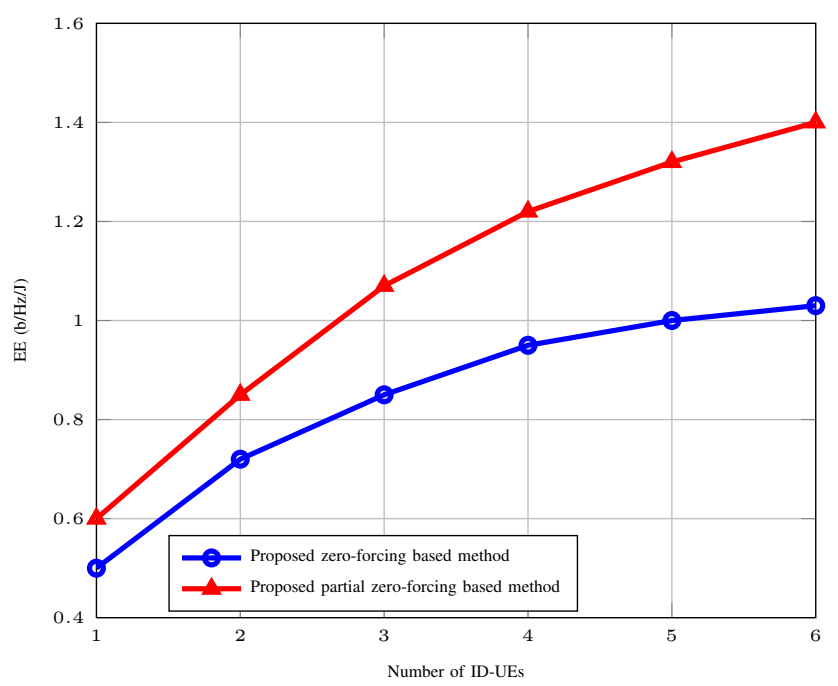

Fig. 6: Performance of the proposed ZF- and PZF-based approaches (EE vs number of ID-UEs).

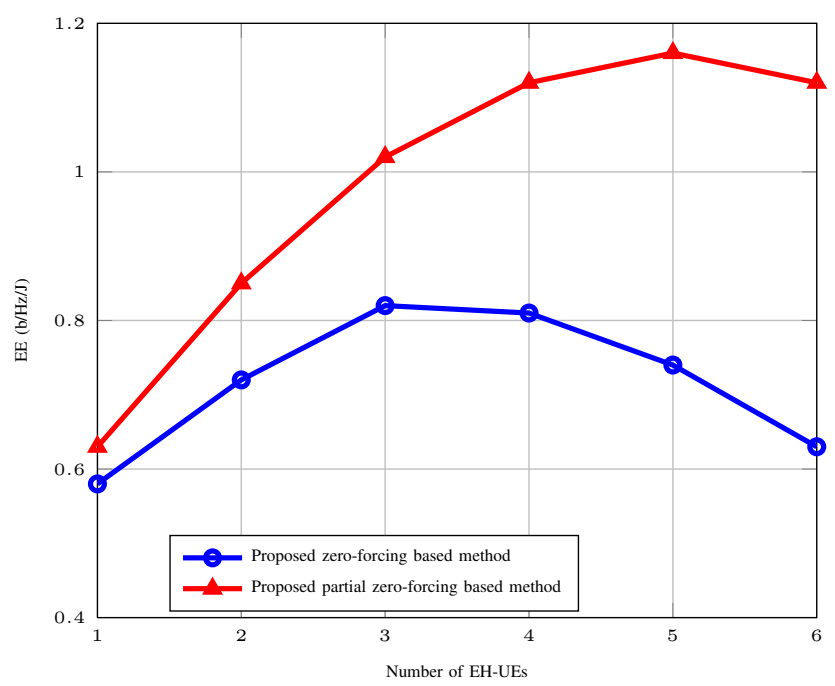

Fig. 7: Performance of the proposed ZF- and PZF-based approaches (EE vs number of EH-UEs).

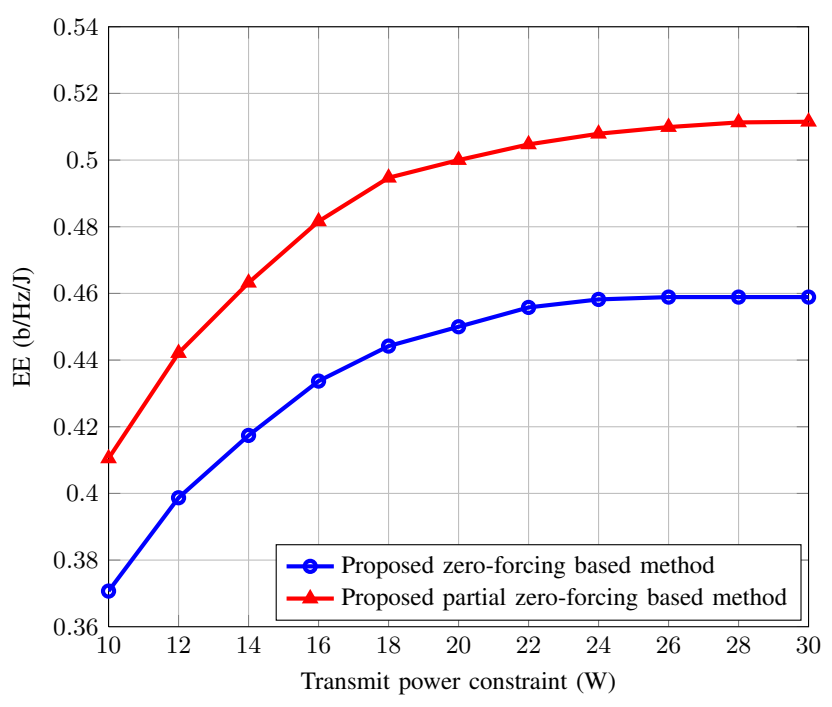

Fig. 8: Performance of the proposed ZF- and PZF-based approaches (EE vs transmit power constraint).

settings: number of small cells, number of ID-UEs, and number of EH-UEs. In this part, we fix the transmit antennas $N_{l}$ in cell $l \in \mathcal{L}$ to 32 . We first investigate the EE performance of the proposed solutions with different numbers of smallcells. As shown in Fig. 5, the optimal EE achieved by both the proposed ZF-based and PZF-based schemes are monotonically non-decreasing. Particularly, for ZF-based solution, the EE increases dramatically with a smaller number of small-cells, i.e., $L<5$, and then approaches an asymptotic value. This is because as the number of transmit antennas is set to 32 , and with 2 ID-UEs and 2 EH-UEs in each cell, the maximum spatial gain is achieved when $L=6$, and thus an asymptotic value exists for the $\mathrm{ZF}$ scheme. On the contrary, with the exploitation of surrounding interference, the EE achieved by the proposed PZF solution increases dramatically even with larger network size. Similar observation has been found when we study the impact of the number of ID-UEs, as shown in Fig. 6. In this simulation, we fix the number of small-cells and EHUEs to 3 and 2, respectively. For the proposed ZF approach, a similar asymptotic value exists for the case of 6 ID-UEs, which is also due to the saturated spatial gain. Although these asymptotic values can be achieved by both cases, the increased number of ID-UEs will consume more resources, which leads to a lower achievable EE. In addition, we have also studied the impact of EH-UEs' number on the EE, where the number of ID-UEs is set to 2. As shown in Fig. 7, the EE first increases then decreases with the increasing number of EH-UEs, and the optimal value is 3 and 5 respectively. This infers that satisfying the minimum EH constraint for larger number of EH-UEs will increase the burden of the system, and hence reduce the EE.

In the next simulation, the EE performance of the proposed ZF-based and PZF-based schemes with various QoS demands are evaluated and shown in Fig. 8, Fig. 9 and Fig. 10. We first investigate the EE performance of the proposed solutions with different power budgets. As shown in Fig. 8, the optimal EE achieved by both the proposed ZF-based and PZF-based schemes are monotonically non-decreasing in the 


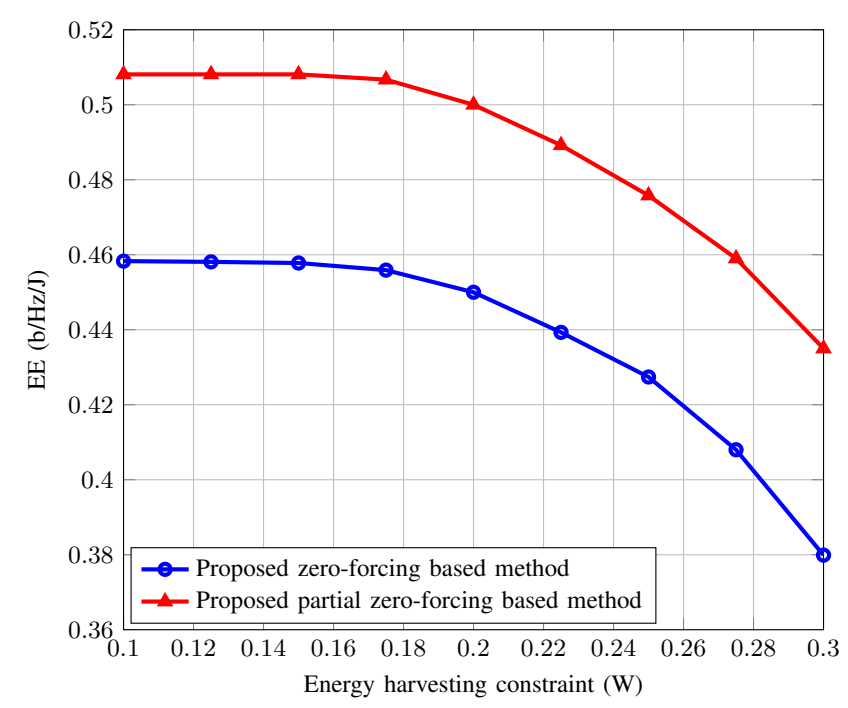

Fig. 9: Performance of the proposed ZF- and PZF-based approaches (EE vs EH constraint).

total transmit power constraint per cell $P_{\max }^{[l]}$. Particularly, the EE increases dramatically with a smaller power budget, i.e., $10<P_{\max }<24 \mathrm{~W}$, and then approaches an asymptotic value where a balance among the achievable rates and the total power consumption is obtained. In other words, the additional power budget does not constitute to extra gain in $\mathrm{EE}$, and similar results are observed in the conventional single-cell systems [46], multi-cell systems [47], and two-tier HetNets [20]. We next show in Fig. 9 and Fig. 10 the optimal EE under different rate and harvested energy requirements. We can observe that the maximum EE remains unchanged up to a particular minimum harvested energy constraint, but decreases thereafter. To satisfy a higher minimum harvested energy demand, extra power is required. It has been shown in Fig. 8 that this additional power cannot provide any EE gain, but in fact, it will weaken the EE performance due to the smaller transmit power range. More precisely, this EE loss is mainly due to increased energy allocated to EH-UEs, which leads to an imbalance between the numerator (transmission rate) and the denominator (power consumption) of the EE metric. A similar trend can also be seen for the case of varying the minimum rate constraint, where the optimal $\mathrm{EE}$ achieved by the proposed ZF-based and PZF-based approaches remains unchanged up to a certain minimum transmission rate requirement, but decreases thereafter. This is because when the minimum rate requirement is low, the required transmit power is also low. Therefore, the most energy efficient design is to operate with a larger transmit power region in order to achieve the optimal EE.

Finally, the effect of the number of transmit antennas on the EE in the proposed CoMP-SWIPT HetNet has been studied. In order to depict the EE gain of the proposed schemes, we compare the performance with mixed beamforming strategy (MBF) that optimize the EE in SWIPT-enabled HetNet [33]. In order to depict the EE gain of SWIPT technology, we compare the performance with approaches that optimize the EE in a conventional two-tier HetNet (without EH capability).

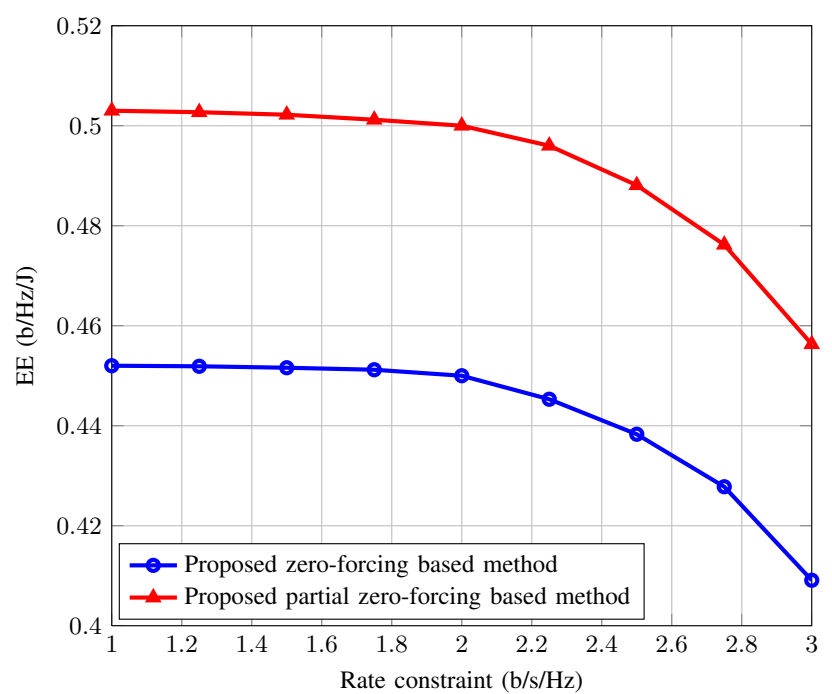

Fig. 10: Performance of the proposed ZF- and PZF-based approaches (EE vs rate constraint).

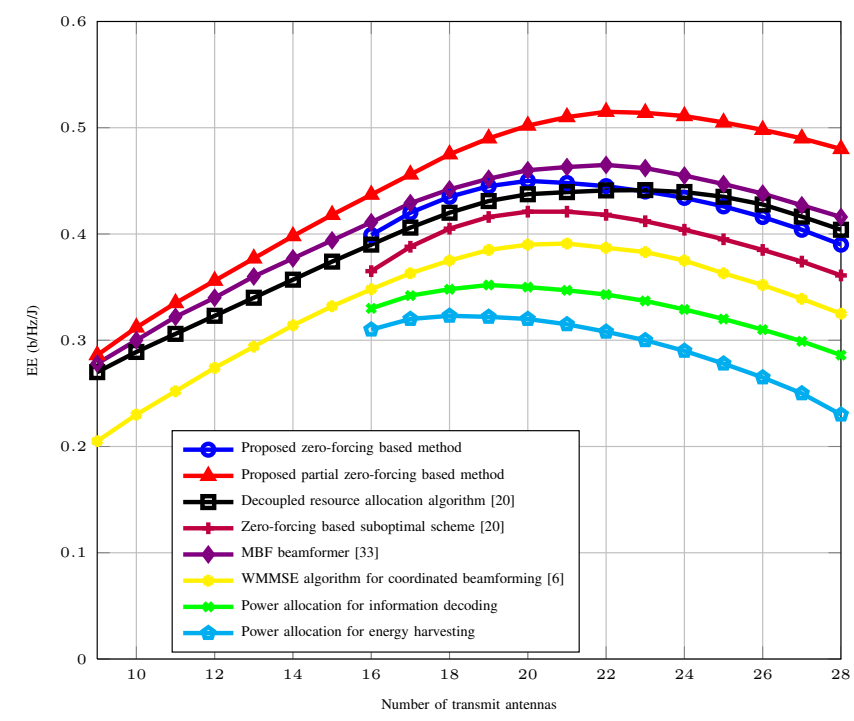

Fig. 11: Impact of the number of transmit antennas on the EE performance under different resource allocation approaches $(L=3$, $K_{l}^{I}=2, K_{l}^{E}=2$ ).

In particular, we take into account the approach with joint transmit beamforming design and power allocation policy [20], and the ZF based suboptimal scheme [20]. Besides, we also compare the performance with approaches that optimize the sum rate using $\mathrm{CB}$ [6]. In addition, the proposed ZF based approach without power allocation is also employed. That is to say, this approach uses all the remaining power (after satisfying the QoS constraints) for either ID or EH. We first investigate the case of $L=3, K_{l}^{I}=2, K_{l}^{E}=2$, where the minimum number of antennas in cell $l$ should be 16 for ZFbased solution and 9 for PZF-based solution. As shown in Fig. 11, our proposed PZF-based solution obtains a superior EE versus the MBF strategy in [33]. This is because the impact of both ID and EH on EE has been considered in our work, and hence these two factors are jointly optimized in order 


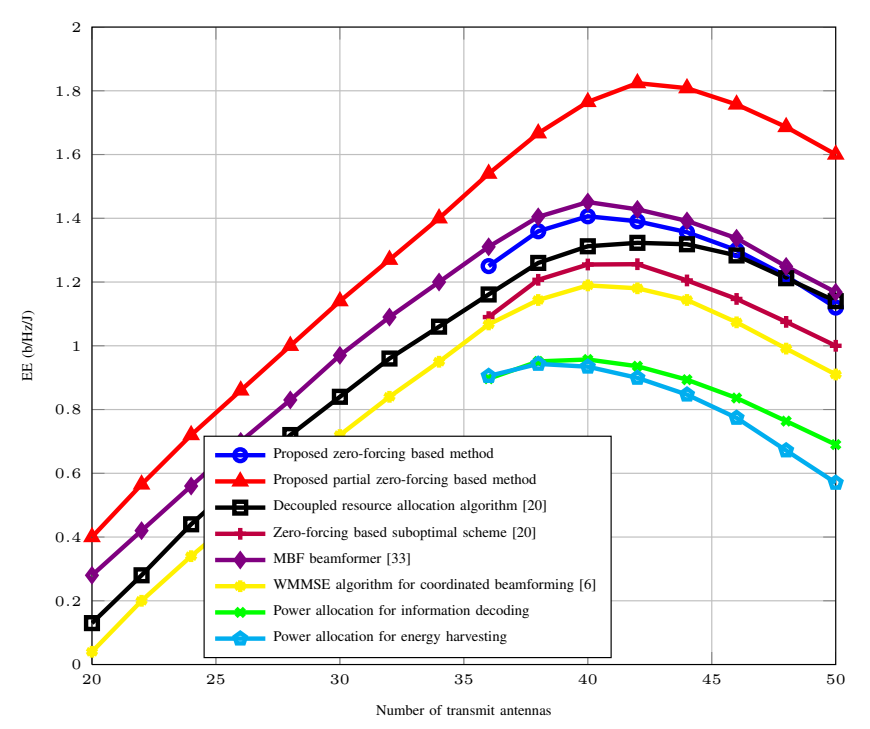

Fig. 12: Impact of the number of transmit antennas on the EE performance under different resource allocation approaches $(L=5$, $K_{l}^{I}=3, K_{l}^{E}=3$ ).

to maximize the EE. In addition, our proposed PZF-based solution achieves a higher EE compared to the approaches that optimize the EE without EH capability [20], and this confirms the advantages of integration SWIPT into CoMP. Specifically, the EE first increases then decreases with the increasing number of transmit antennas, and the optimal value is 22. This is because activating more transmit antennas will offer extra signal spaces for transmitting data as well as energy, and hence, this is beneficial for increasing the EE. At the same time, activating more transmit antennas will cost additional circuit power, resulting in some loss of EE. In other words, a saturation occurs when $N_{l}=22$, and this can also be observed in the conventional single-cell systems [46]. Interestingly, in the case with a large number of transmit antennas, i.e., $N_{l} \geq 23$, the EE achieved by the scheme without EH capability is even higher than that of the proposed ZF-based scheme. This infers that the EH-provided EE gain cannot compensate the extra cost of activating redundant antennas, and this can also be observed for a SWIPT-based point-to-point MIMO system [32]. It should be noted that the proposed PZF scheme can achieve a similar EE compared to its opponents, but with less number of transmit antennas. For instance, to achieve $0.4 \mathrm{bits} / \mathrm{Hz} / \mathrm{J}$, the required transmit antennas for the proposed PZF scheme is 14. However, this number is increased to $16,17,18$ and 19 for the proposed $\mathrm{ZF}$ scheme, decoupled resource allocation scheme [20], ZF suboptimal approach [20], respectively, and this has proved the practicality of our proposed approaches. At last, the scheme that uses all the remaining resources for EH is the least energyefficient strategy, and this trend worsens with larger number of antennas. With a larger system configuration, i.e., $L=5$, $K_{l}^{I}=3, K_{l}^{E}=3$, as shown in Fig. 12, a similar trend can be observed. Specifically, an increased EE gain can be provided by the proposed ZF and PZF approaches, and this has proved the applicability of our proposed approaches.

\section{CONCLUSIONS}

We studied the problem of QoS-constrained EE maximization in the downlink of CoMP-SWIPT HetNets. The corresponding optimization problem in the presence of inter- and intra-cell interference leads to a non-convex problem, and thus the solution is non-trivial and cannot be obtained directly. To solve this problem, a decoupled algorithm has been proposed where the beamforming design and power allocation procedure are separated. In particular, two strategies based on the idea of $\mathrm{ZF}$ beamforming were devised to handle the complicated intraand inter-cell interference. In the first proposed approach, the considered HetNet scenario was transformed to a virtual pointto-point system via canceling all the interference to both IDUEs and EH-UEs. An efficient solution was then developed to maximize the EE. On the other hand, the ZF strategy does not utilize the notion that interference benefits the EH-UEs. As a result, we propose a PZF approach by differentiating the EHUEs and ID-UEs in order to further improve the EE. Through constructing null spaces, a hybrid transmit beamforming was then developed to optimally combine the column spaces towards maximizing EE. Our findings illustrated that the EE can be improved considerably through integration of CoMPSWIPT technology in HetNets.

\section{APPENDIX A}

\section{PROOF OF PROPOSITION 1}

To prove Proposition 1, $\lambda_{E E}$ is first reformulated as (71) in the next page. Suppose EH-UE $\{\hat{k}, \hat{l}\} \in\left\{\mathcal{K}_{l}^{E}, \mathcal{L}\right\}$ satisfies the constraint $\eta \tilde{g}_{[\hat{k}, \hat{l}]}>\zeta$, and $\lambda_{E E}^{*}\left(P_{T}\right)$ represents the maximum achievable energy efficiency with transmit power $P_{T}$. Hence, with the incremental power $\triangle p$ allocated to the EH-UE $\{\hat{k}, \hat{l}\}$, we obtain (72) in the next page. Thus, the maximum EE $\lambda_{E E}$ is a non-decreasing function with the increasing transmit power $P_{T}$. This completes the proof.

\section{APPENDIX B}

\section{Proof of Proposition 2}

To prove $\lambda_{E E}^{*}\left(p_{[k, l]}\right)$ is quasiconcave, the superlevel sets of $\lambda_{E E}^{*}\left(p_{[k, l]}\right)$ are written as (73) in the next page. Thus, if $\mathcal{S}_{\pi}$ is convex for any real number $\pi$, the quasiconcavity of $\lambda_{E E}^{*}\left(p_{[k, l]}\right)$ holds [39]. Hence, the proof can be divided into separate cases, For $\pi<0$, considering that the EE is strictly positive $\left(\lambda_{E E}>0\right)$, there aren't any points on the counter, $\lambda_{E E}^{*}\left(p_{[k, l]}\right)=\pi$. Furthermore, for $\pi \geq 0$, we can reformulate $\lambda_{E E}$ using

$$
\lambda_{E E}=\frac{C\left(p_{[k, l]}\right)}{L\left(p_{[k, l]}\right)+M},
$$

where $C\left(p_{[k, l]}\right)$ denotes the capacity of the system, $L\left(p_{[k, l]}\right)$ is a linear function of $p_{[k, l]}$ and $L\left(p_{[k, l]}\right)=\sum_{l \in \mathcal{L}} \sum_{k \in \mathcal{K}_{l}^{I E}}[\zeta-$ $\left.\eta\left(1-\alpha_{[k, l]}\right) \bar{h}_{[k, l]}\right] p_{[k, l]}$, and $M=\sum_{l \in \mathcal{L}} P_{a n t} N_{l}+P_{l}^{s t a}$ denotes a constant. Hence, $\mathcal{S}_{\pi}$ is equivalent to $\pi L\left(p_{[k, l]}\right)+\pi M-$ $C\left(p_{[k, l]}\right) \leq 0$. Given that $C\left(p_{[k, l]}\right)$ is concave in regard to $p_{[k, l]}[39]$, and $\pi L\left(p_{[k, l]}\right)$ is linear over $p_{[k, l]}, \mathcal{S}_{\pi}$ is convex and thus $\lambda_{E E}^{*}\left(p_{[k, l]}\right)$ is strictly quasi-concave with respect to $p_{[k, l]}$. This concludes the proof of Proposition 2. 


$$
\begin{aligned}
\lambda_{E E} & =\frac{\sum_{l \in \mathcal{L}} \sum_{k \in \mathcal{K}_{l}^{I}} W \log _{2}\left(1+p_{[k, l]} \tilde{h}_{[k, l]}\right)}{\sum_{l \in \mathcal{L}}\left[\zeta\left(\sum_{k \in \mathcal{K}_{l}^{I}} p_{[k, l]}+\sum_{k \in \mathcal{K}_{l}^{E}} q_{[k, l]}\right)+P_{a n t} N_{l}+P_{l}^{s t a}-\sum_{k \in \mathcal{K}_{l}^{E}} \eta q_{[k, l]} \tilde{g}_{[k, l]}\right]} \\
& =\frac{\sum_{l \in \mathcal{L}} \sum_{k \in \mathcal{K}_{l}^{I}} W \log _{2}\left(1+p_{[k, l]} \tilde{h}_{[k, l]}\right)}{\sum_{l \in \mathcal{L}}\left[\zeta \sum_{k \in \mathcal{K}_{l}^{I}} p_{[k, l]}+P_{a n t} N_{l}+P_{l}^{s t a}-\sum_{k \in \mathcal{K}_{l}^{E}}\left(\eta \tilde{g}_{[k, l]}-\zeta\right) q_{[k, l]}\right]}
\end{aligned}
$$

$$
\lambda_{E E}\left(P_{T}+\triangle p\right)=\frac{\sum_{l \in \mathcal{L}} \sum_{k \in \mathcal{K}_{l}^{I}} W \log _{2}\left(1+p_{[k, l]} \tilde{h}_{[k, l]}\right)}{\sum_{l \in \mathcal{L}}\left[\zeta \sum_{k \in \mathcal{K}_{l}^{I}} p_{[k, l]}+\hat{P}_{l}-\sum_{k \in \mathcal{K}_{l}^{E}}\left(\eta \tilde{g}_{[k, l]}-\zeta\right) q_{[k, l]}\right]-\left(\eta \tilde{g}_{[\hat{k}, \hat{l}]}-\zeta\right) \triangle p} \geq \lambda_{E E}^{*}\left(P_{T}\right)
$$

$$
\mathcal{S}_{\pi}=\left\{\sum_{k \in \mathcal{K}_{l}^{I E}} p_{[k, l]} \leq P_{\text {max }}^{[l]} ; \delta_{[k, l]}^{-1} \leq p_{[k, l]} ; \rho_{[k, l]}^{-1} \leq p_{[k, l]} \mid \lambda_{E E}^{*}\left(p_{[k, l]}\right) \geq \pi\right\}
$$

\section{REFERENCES}

[1] H. Zhang, Y. Dong, J. Cheng, M. J. Hossain, and V. C. M. Leung, "Fronthauling for 5G LTE-U ultra dense cloud small cell networks," IEEE Wireless Commun., vol. 23, no. 6, pp. 48 - 53, Dec. 2016.

[2] J. G. Andrews, "Seven ways that HetNets are a cellular paradigm shift," IEEE Commun. Mag., vol. 51, no. 3, pp. 136-144, Mar. 2013.

[3] A. Shojaeifard, K. A. Hamdi, E. Alsusa, D. K. C. So, and J. Tang, "A unified model for the design and analysis of spatially-correlated loadaware HetNets," IEEE Trans. Commun., vol. 62, no. 11, pp. 1-16, Nov. 2014.

[4] M. K. Karakayali, G. J. Foschini, and R. A. Valenzuela, "Network coordination for spectrally efficient communications in cellular systems," IEEE Wireless Commun. Mag., vol. 13, no. 4, pp. 56 - 61, Aug. 2006.

[5] C. Yang, S. Han, X. Hou, and A. F. Molisch, "How to design CoMP to achieve its promised potential?" IEEE Wireless Commun. Mag., vol. 20, no. 1, pp. 67 - 74, Feb. 2013.

[6] Q. Shi, M. Razaviyayn, Z. Luo, and C. He, "An iteratively weighted MMSE approach to distributed sum-utility maximization for a MIMO interfering broadcast channel," IEEE Trans. on Sig. Process., vol. 59, no. 9 , pp. $4331-4340$, Sep. 2011.

[7] S. Mosleh, L. Liu, and J. Zhang, "Proportional-fair resource allocation for coordinated multi-point transmission in LTE-advanced," IEEE Transaction on Wireless Communications, vol. 15, no. 8, pp. 5355 - 5367 , Aug. 2016.

[8] J. C. H. Chen, H. Zhang, and F. Zhao, "Evolution of limited-feedback CoMP systems from 4G to 5G," IEEE Veg. Tech. Mag., vol. 9, no. 3, pp. $94-103$, Sep. 2014.

[9] Q. Cui, H. Wang, P. Hu, X. Tao, P. Zhang, J. Hamalainen, and L. Xia, "Evolution of limited-feedback comp systems from 4G to 5G: CoMP features and limited-feedback approaches," IEEE Veh. Tech. Mag., vol. 9, no. 3, pp. $94-103$, Sep. 2014.

[10] V. Jungnickel, K. Manolakis, W. Zirwas, B. Panzner, V. Braun, M. Lossow, M. Sternad, R. Apelfröjd, and T. Svensson, "The role of small cells, coordinated multipoint, and massive MIMO in 5G," IEEE Commun. Mag., vol. 52, no. 5, pp. 44 - 51, May 2014.

[11] T. Zhang, H. Fan, J. Loo, and D. Liu, "User preference aware caching deployment for device-to-device caching networks," IEEE Systems Journal, doi: 10.1109/JSYST.2017.2773580.

[12] C. Xiong, G. Li, S. Zhang, Y. Chen, and S. Xu, "Energy- and spectralefficiency tradeoff in downlink OFDMA networks," IEEE Trans. Wireless Commun., vol. 10, no. 11, pp. 3874-3886, Nov. 2011.

[13] H. Zhang, C. Jiang, N. Beaulieu, X. Chu, X. Wen, and M. Tao, "Resource allocation in spectrum-sharing OFDMA femtocells with heterogeneous services," IEEE Trans. on Commun., vol. 62, no. 7, pp. 2366 - 2377, July 2014.

[14] N. Zhao, F. R. Yu, and H. Sun, "Adaptive energy-efficient power allocation in green interference-alignment-based wireless networks," IEEE Trans. on Veh. Tech., vol. 64, no. 9, pp. 4268 - 4281, Sep. 2015.

[15] J. Tang, D. K. C. So, E. Alsusa, K. Hamdi, A. Shojaeifard, and K. K. Wong, "Energy-efficient heterogeneous cellular networks with spectrum underlay and overlay access," to appear in IEEE Trans. on Veh. Tech., 2017.
[16] G. Lim, C. Xiong, L. J. C. Jr., and G. Y. Li, "Energy-efficient resource allocation for OFDMA-based multi-RAT networks," IEEE Trans. Wireless Commun., vol. 13, no. 5, pp. 2696- 2705, May 2014.

[17] R. Ramamonjison and V. K. Bhargava, "Energy efficiency maximization framework in cognitive downlink two-tier networks," IEEE Trans. Wireless Commun., vol. 14, no. 3, pp. 1468- 1479, Mar. 2015.

[18] H. Zhang, Y. Nie, J. Cheng, V. C. M. Leung, and A. Nallanathan, "Sensing time optimization and power control for energy efficient cognitive small cell with imperfect hybrid spectrum sensing," IEEE Trans. on Wireless Commun., vol. 16, no. 2, pp. 730 - 743, Feb. 2017.

[19] Z. Xu, C. Yang, G. Y. Li, Y. Liu, and S. Xu, "Energy-efficient CoMP precoding in heterogeneous networks," IEEE Trans. Sig. Process., vol. 62, no. 4, pp. 1005 - 1017, Feb. 2014.

[20] J. Tang, D. So, E. Alsusa, K. A. Hamdi, and A. Shojaeifard, "Resource allocation for energy efficiency optimization in heterogeneous networks," IEEE Journal on Sel. Areas in Commun., vol. 33, no. 10, pp. 2104 2117, Oct. 2015.

[21] X. Lu, P. Wang, D. Niyato, D. I. Kim, and Z. Han, "Wireless networks with RF energy harvesting: A contemporary survey," Commun. Surveys Tuts., vol. 17, no. 2, pp. 757-789, 2nd Quart. 2015.

[22] N. Zhao, F. R. Yu, and V. C. M. Leung, "Wireless energy harvesting in interference alignment networks," IEEE Commun. Mag., vol. 53, no. 6, pp. $72-78$, June 2015.

[23] — , "Opportunistic communications in interference alignment networks with wireless power transfer," IEEE Wireless Commun., vol. 22, no. 1 , pp. 88 - 95, March 2015.

[24] L. R. Varshney, "Transporting information and energy simultaneously," in Proc. 2008 IEEE Int. Symp. Inf. Theory, July 2008, pp. 1612-1616.

[25] E. Hossain, M. Rasti, H. Tabassum, and A. Abdelnasser, "Evolution toward 5G multi-tier cellular wireless networks: An interference management perspective," IEEE Trans. Wireless Commun., vol. 21, no. 3, pp. 118-127, June 2014.

[26] J. Tang, D. K. C. So, A. Shojaeifard, and K. K. Wong, "Energy efficiency optimization with SWIPT in MIMO broadcast channels for internet of things," IEEE Internet of Things Journal, vol. 5, no. 4, pp. 2605 - 2619, Aug. 2018.

[27] R. Zhang and C. K. Ho, "MIMO broadcasting for simultaneous wireless information and power transfer," IEEE Trans. Wireless Commun., vol. 12, no. 5, pp. 1989-2001, May 2013.

[28] S. Timotheou, I. Krikidis, S. Karachontzitis, and K. Berberidis, "Spatial domain simultaneous information and power transfer for MIMO channels," IEEE Trans. Wireless Commun., vol. 14, no. 8, pp. 4115-4128, Aug. 2015.

[29] D. W. K. Ng, E. S. Lo, and R. Schober, "Wireless information and power transfer: energy efficiency optimization in OFDMA systems," IEEE Trans. Wireless Commun., vol. 12, no. 12, pp. 6352-6370, Dec. 2013.

[30] Q. Shi, C. Peng, W. Xu, and M. Hong, "Energy efficiency optimization for MISO SWIPT systems with zero-forcing beamforming," IEEE Trans. Sig. Process., vol. 64, no. 4, pp. 842-854, Feb. 2016.

[31] S. He, Y. Huang, W. Chen, S. Jin, H. Wang, and L. Yang, "Energy efficient coordinated precoding design for a multicell system with RF energy harvesting," EURASIP J. Wireless Commun. Netw., no. 1, pp. 1-12, Mar. 2015.

[32] J. Tang, D. K. C. So, A. Shojaeifard, K. K. Wong, and J. Wen, "Joint antenna selection and spatial switching for energy efficient MIMO 
SWIPT system," IEEE Trans. on Wireless Commun., vol. 16, no. 7, pp. $4754-4769$, July 2017.

[33] M. Sheng, L. Wang, X. Wang, Y. Zhang, C. Xu, and J. Li, "Energy efficient beamforming in miso heterogeneous cellular networks with wireless information and power transfer," IEEE J. Sel. Areas Commun., vol. 34, no. 4, pp. 954 - 968, Apr. 2016.

[34] D. W. K. Ng, E. Lo, and R. Schober, "Energy-efficient resource allocation in multiuser OFDM systems with wireless information and power transfer," in Proc. IEEE Wireless Commun. and Netw. Conf. (WCNC), 2013.

[35] _ - "Wireless information and power transfer: energy efficiency optimization in OFDMA systems," IEEE Trans. Wireless Commun., vol. 12, no. 12, pp. 6352-6370, Dec. 2013.

[36] J. C. H. Chen, H. Zhang, and F. Zhao, "Spectral-energy efficiency tradeoff in relay-aided massive MIMO cellular networks with pilot contamination," IEEE Access, vol. 4, pp. 5234 - 5242, Sep. 2016.

[37] L. Zhang, Y. Xin, and Y. C. Liang, "Weighted sum rate optimization for cognitive radio MIMO broadcast channels," IEEE Trans. Wireless Commun., vol. 8, no. 9, pp. 2950-2959, Jun. 2009.

[38] W. Dinkelbach, "On nonlinear fractional programming," Management Science, vol. 13, pp. 492-498, Mar. 1967.

[39] S. Boyd and L. Vandenberghe, Convex Optimization. Cambridge University Press, Cambridge, UK, 2004.

[40] W. Yu, W. Rhee, S. Boyd, and J. M. Cioffi, "Iterative water-filling for Gaussian vector multiple-access channels," IEEE Trans. on Inf. Theory, vol. 50, no. 1, pp. 145-152, Jan. 2004.
[41] N. Jindal, W. Rhee, S. Vishwanath, S. A. Jafar, and A. Goldsmith, "Sum power iterative water-filling for multi-antenna gaussian broadcast channels," IEEE Trans. Inform. Theory, vol. 51, no. 4, pp. 1570 - 1580, Apr. 2005.

[42] W. Yu, "Sum-capacity computation for the gaussian vector broadcast channel via dual decomposition," IEEE Trans. Inform. Theory, vol. 52, no. 2, pp. $754-759$, Feb. 2006.

[43] I. Krikidis, S. Timotheou, S. Nikolaou, G. Zheng, D. W. K. Ng, and R. Schober, "Simultaneous wireless information and power transfer in modern communication systems," IEEE Commun. Mag., vol. 52, no. 11, pp. 104-110, Nov. 2014

[44] IEEE P802.11 Wireless LANs, "TGn Channel Models", IEEE 802.1103/940r4, Tech. Rep., May 2004.

[45] E. Björnson, L. Sanguinetti, J. Hoydis, and M. Debbah, "Optimal design of energy-efficient multi-user mimo systems: Is massive MIMO the answer?" IEEE Trans. Wireless Commun., vol. 14, no. 6, pp. 3059 3075, June 2015.

[46] J. Tang, D. K. C. So, E. Alsusa, K. A. Hamdi, and A. Shojaeifard, "On the energy efficiency-spectral efficiency trade-off in MIMO-OFDMA broadcast channels," IEEE Trans. Veh. Tech., vol. 65, no. 7, pp. 51855199, July 2016.

[47] J. Tang, D. So, E. Alsusa, K. A. Hamdi, and A. Shojaeifard, "Energy efficiency optimization with interference alignment in multi-cell MIMO interfering broadcast channels," IEEE Trans. Commun., vol. 63, no. 7, pp. 2486-2499, July 2015. 